\title{
Gravitational instability of isothermal and polytropic spheres
}

\author{
P. H. Chavanis ${ }^{\star}$
}

\author{
Laboratoire de Physique Quantique, Université Paul Sabatier, 118 route de Narbonne, 31062 Toulouse, France
}

Received 3 July 2002 / Accepted 19 November 2002

\begin{abstract}
We complete previous investigations on the thermodynamics of self-gravitating systems by studying the grand canonical, grand microcanonical and isobaric ensembles. We also discuss the stability of polytropic spheres in connexion with a generalized thermodynamical approach proposed by Tsallis. We determine in each case the onset of gravitational instability by analytical methods and graphical constructions in the Milne plane. We also discuss the relation between dynamical and thermodynamical stability of stellar systems and gaseous spheres. Our study provides an aesthetic and simple approach to this otherwise complicated subject.
\end{abstract}

Key words. stellar dynamics - hydrodynamics - instabilities - gravitation

\section{Introduction}

The statistical mechanics of systems interacting via longrange forces exhibit peculiar features such as negative specific heats, inequivalence of statistical ensembles and phase transitions. These curious behaviours have been first discussed in the astrophysical literature during the elaboration of a thermodynamics for stars (Eddington 1926), globular clusters (Lynden-Bell \& Wood 1968), black holes (Hawking 1974) and galaxies (Padmanabhan 1990). They have been recently rediscovered in different fields of physics such as nuclear physics, plasma physics, Bose-Einstein condensates, atomic clusters, two-dimensional turbulence... The main challenge is represented by the construction of a thermodynamic treatment of systems with long-range forces and by the analogies and differences among the numerous domains of application (see Chavanis 2002e and other contributions in that book).

Gravity provides a fundamental example of unshielded long-range interaction for which ideas of statistical mechanics and thermodynamics can be developed and tested. For systems with long-range interactions, the mean-field approximation is known to be exact in a suitable thermodynamic limit. Therefore, the structure and stability of self-gravitating systems at statistical equilibrium can be analyzed in terms of the maximization of a thermodynamical potential. This thermodynamical approach leads to isothermal configurations which have been studied for a long time in the context of stellar structure (Chandrasekhar 1942) and galactic structure (Binney \& Tremaine 1987). As is well-known, isothermal spheres have infinite mass so that the system must be confined within a box (Antonov problem) in order to prevent evaporation and make the thermodynamical approach rigorous. It is also well-known that isothermal configurations only correspond to metastable

\footnotetext{
* e-mail: chavanis@irsamc2.ups-tlse.fr
}

equilibrium states (i.e., local maxima of the thermodynamical potential), not true equilibrium states. These metastable equilibrium states are expected to be relevant, however, for the timescales contemplated in astrophysics. In particular, globular clusters described by Michie-King models are probably in such metastable states.

The series of equilibria of finite isothermal spheres can be parametrized by the density contrast between the center and the boundary of the system. For sufficiently low density contrasts, the system is thermodynamically stable. However, instability occurs at sufficiently large concentrations: at some point in the series of equilibria, the solutions cease to be local maxima of the thermodynamical potential and become unstable saddle points. The crucial point to realize is that the onset of instability depends on the statistical ensemble considered: microcanonical (MCE), canonical (CE) or grand canonical (GCE). This contrasts with ordinary systems, with short range interactions, for which the statistical ensembles are equivalent at the thermodynamic limit, except near a phase transition. This suggests that gravitating systems in virial equilibrium are similar to normal (extensive) systems at the verge of a phase transition (Padmanabhan 1990).

The thermodynamical stability of self-gravitating systems can be studied by different technics. Horwitz \& Katz (1978) use a field theory and write the density of states, the partition function and the grand partition function in MCE, CE and GCE respectively as a path integral for a formal field $\phi$. In the meanfield approximation, the integral is dominated by the distribution $\phi_{0}$ which maximizes a specific action $A[\phi]$. In the grand canonical ensemble, $A_{G C E}[\phi]$ is the Liouville action. Horwitz \& Katz (1978) solve the problem numerically and find that the series of equilibria become unstable for a density contrast $1.58,32.1$ and 709 in GCE, CE and MCE respectively. It is normal that the critical density contrast (hence the stability of the 
system) increases when more and more constraints are added on the system (conservation of mass in $\mathrm{CE}$, conservation of mass and energy in MCE). This field theory has been rediscussed recently by de Vega \& Sanchez (2002) who confirmed previous results and proposed interesting developements.

The thermodynamical stability of self-gravitating systems can also be settled by studying whether an isothermal sphere is a maximum or a saddle point of an appropriate thermodynamical potential: the entropy in MCE, the free energy in CE and the grand potential in GCE. The change of stability can be determined very easily from the topology of the equilibrium phase diagram by using the turning point criterion of Katz (1978) who has extended Poincaré's theory on linear series of equilibria (see also Lynden-Bell \& Wood 1968). This method is very powerful but it does not provide the form of the perturbation profile that triggers the instability. This perturbation profile can be obtained by computing explicitly the second order variations of the thermodynamical potential and reducing the problem of stability to the study of an eigenvalue equation. This study was first performed by Antonov (1962) in MCE and revisited by Padmanabhan (1989) with a simpler mathematical treatment. This analysis was extended in CE by Chavanis (2002a) who showed in addition the equivalence between thermodynamical stability and dynamical stability with respect to Navier-Stokes equations (Jeans problem). Remarkably, this stability analysis can be performed analytically by using simple graphical constructions in the Milne plane.

In the present paper, we propose to extend these analytical methods to more general situations in order to provide a complete description of the thermodynamics of spherical selfgravitating systems. In Sect. 2, we review the stability limits of isothermal spheres in different ensembles by using the turning point criterion. In Sect. 3, we consider specifically the grand canonical and grand microcanonical ensembles and evaluate the second order variations of the associated thermodynamical potential. In Sect. 4, we briefly discuss the connexion between thermodynamics and statistical mechanics (and field theory) for self-gravitating systems. In Sect. 5, we consider the case of generalized thermodynamics proposed by Tsallis (1988) and leading to stellar polytropes (Plastino \& Plastino 1997; Taruya \& Sakagami 2002a,b; Chavanis 2002b). In Sect. 6, we discuss the stability of isothermal and polytropic spheres under an external pressure (Bonnor problem). We provide a new and entirely analytical solution of this old problem. Finally, in Sect. 7 we discuss the relation between dynamical and thermodynamical stability of stellar systems and gaseous spheres.

\section{Thermodynamics of self-gravitating systems}

\subsection{Thermodynamical ensembles}

Consider a system of $N$ particles, each of mass $m$, interacting via Newtonian gravity. Let $f(\boldsymbol{r}, \boldsymbol{v}, t)$ denote the distribution function of the system and $\rho(\boldsymbol{r}, t)=\int f \mathrm{~d}^{3} \boldsymbol{v}$ the spatial density. The total mass and total energy can be expressed as

$M=\int \rho \mathrm{d}^{3} \boldsymbol{r}$
$E=\frac{1}{2} \int f v^{2} \mathrm{~d}^{3} \boldsymbol{r} \mathrm{d}^{3} \boldsymbol{v}+\frac{1}{2} \int \rho \Phi \mathrm{d}^{3} \boldsymbol{r}=K+W$,

where $K$ is the kinetic energy and $W$ the potential energy. The gravitational potential $\Phi$ is related to the spatial density by the Poisson equation

$\Delta \Phi=4 \pi G \rho$.

The Boltzmann entropy is given by the standard formula (within an additional constant term)

$S=-k \int\left\{\frac{f}{m} \ln \frac{f}{m}-\frac{f}{m}\right\} \mathrm{d}^{3} \boldsymbol{r} \mathrm{d}^{3} \boldsymbol{v}$,

which can be obtained by a combinatorial analysis (Ogorodnikov 1965). For the moment, we shall assume that the system is confined within a spherical box of radius $R$ so that its volume is fixed.

In the microcanonical ensemble, the system is isolated and conserves energy $E$ and particle number $N$ (or mass $M=N m$ ). Statistical equilibrium corresponds to the state that maximizes the entropy $S$ at fixed $E$ and $N$. Introducing Lagrange multipliers $1 / T$ and $-\mu / T$ for each constraint, the first order variations of entropy satisfy the relation

$\delta S-\frac{1}{T} \delta E+\frac{\mu}{T} \delta N=0$,

where $T$ is the temperature and $\mu$ the chemical potential. Equation (5) can be regarded as the first principle of thermodynamics.

In the canonical ensemble, the temperature and the particle number are fixed allowing the energy to fluctuate. In that case, the relevant thermodynamical parameter is the free energy (more precisely the Massieu function) $J=S-\frac{1}{T} E$ which is related to $S$ by a Legendre transformation. According to Eq. (5), the first variations of $J$ satisfy $\delta J=-E \delta\left(\frac{1}{T}\right)-\frac{\mu}{T} \delta N$. Therefore, at statistical equilibrium, the system is in the state that maximizes the free energy $J$ at fixed particle number $N$ and temperature $T$.

In the grand canonical ensemble, both temperature and chemical potential are fixed, allowing the energy and the particle number to fluctuate. The relevant thermodynamical potential is now the grand potential $G=S-\frac{1}{T} E+\frac{\mu}{T} N$. Its first variations satisfy $\delta G=-E \delta\left(\frac{1}{T}\right)+N \delta\left(\frac{\mu}{T}\right)$. At statistical equilibrium, the system is in the state that maximizes $G$ at fixed $T$ and $\mu / T$.

The microcanonical, canonical and grand canonical ensembles are the most popular. However, we are free to define other thermodynamical ensembles. For example, Lecar \& Katz (1981) have introduced a grand microcanonical ensemble (GMCE) in which the fugacity and the energy are fixed. This corresponds to a thermodynamical potential $\mathcal{K}=S+\frac{\mu}{T} N$ whose first variations satisfy $\delta \mathcal{K}=\frac{1}{T} \delta E+N \delta\left(\frac{\mu}{T}\right)$. At statistical equilibrium, the system is in the state that maximizes $\mathcal{K}$ at fixed $E$ and $\mu / T$.

\subsection{The isothermal distribution}

For extensive systems, it is well-known that the statistical ensembles described previously are all equivalent at the thermodynamic limit. Therefore, the choice of a particular ensemble 
(in general the grand canonical one) is only dictated by reasons of convenience. This is not the case for systems with long-range interactions such as gravitational systems. Indeed, the statistical ensembles are not interchangeable and the stability limits differ from one ensemble to the other. Therefore, the choice of the ensemble is imposed by the physical properties of the system under consideration. If we want to describe in terms of statistical mechanics globular clusters (Lynden-Bell \& Wood 1968) and elliptical galaxies (Lynden-Bell 1967; Hjorth \& Madsen 1993; Chavanis \& Sommeria 1998), which can be treated as isolated systems in a first approximation, the good choice is the microcanonical ensemble. If now the system is in contact with a radiation background imposing its temperature, the relevant ensemble is the canonical one. This ensemble may be appropriate to star or galaxy formation (Penston 1969; Chavanis 2002a,b), white dwarf and neutron stars (Hertel \& Thirring 1971; Chavanis 2002c,d) and dark matter made of massive neutrinos (Bilic \& Viollier 1997; Chavanis 2002g). This canonical description is also exact in a model of selfgravitating Brownian particles (Chavanis et al. 2002; Sire \& Chavanis 2002). Finally, the grand canonical ensemble may be appropriate to the interstellar medium (or possibly the largescale structures of the universe), assuming that a statistical description is relevant (de Vega et al. 1998). Possible applications of the grand microcanonical ensemble are discussed by Lecar \& Katz (1981).

If we just cancel the first order variations of the thermodynamical potential (under constraints appropriate to the ensemble considered), it is straightforward to check that each ensemble yields the Maxwell-Boltzmann distribution

$f=m \mathrm{e}^{\mu / k T} \mathrm{e}^{-\frac{m}{k T}\left(\frac{v^{2}}{2}+\Phi\right)}$.

Therefore, the statistical equilibrium state of a self-gravitating system is isothermal whatever the ensemble considered. The differences will come from the second order variations of the thermodynamical potential. The choice of the statistical ensemble will affect the stability of the system.

The density field associated with the distribution function (6) is given by

$\rho=\left(\frac{2 \pi}{\beta}\right)^{3 / 2} z \mathrm{e}^{-\beta \Phi}$,

where we have defined the fugacity $z$ and the inverse temperature $\beta$ by the relations

$z=m \mathrm{e}^{\mu / k T}, \quad \beta=\frac{m}{k T}$.

The distribution function (6) can then be written in terms of $\rho$ as $f=\left(\frac{m}{2 \pi k T}\right)^{3 / 2} \rho(\boldsymbol{r}) \mathrm{e}^{-\frac{m v^{2}}{2 k T}}$.

This distribution function is a global maximum of the thermodynamical potential under the usual constraints and for a given density field $\rho(\boldsymbol{r})$ (see Padmanabhan 1989; Chavanis 2002a). Substituting this optimal distribution in Eqs. (2) and (4), we get $E=\frac{3}{2} N k T+\frac{1}{2} \int \rho \Phi \mathrm{d}^{3} \boldsymbol{r}$,

$\frac{S}{k}=\frac{5 N}{2}+\frac{3 N}{2} \ln \left(\frac{2 \pi k T}{m}\right)-\int \frac{\rho}{m} \ln \frac{\rho}{m} \mathrm{~d}^{3} r$.
It will be more convenient in the sequel to study the stability problem directly from these equations, without loss of generality.

\subsection{The Emden equation and the Milne variables}

Inserting the relation (7) in Eq. (3), we find that the gravitational potential $\Phi$ is a solution of the Boltzmann-Poisson equation

$\Delta \Phi=4 \pi G A \mathrm{e}^{-\beta \Phi}$.

If we introduce the function $\psi=\beta\left(\Phi-\Phi_{0}\right)$ where $\Phi_{0}$ is the gravitational potential at $r=0$, the density field can be written

$\rho=\rho_{0} \mathrm{e}^{-\psi}$,

where $\rho_{0}$ is the central density. Introducing the normalized distance $\xi=\left(4 \pi G \beta \rho_{0}\right)^{1 / 2} r$, the Boltzmann-Poisson equation can be written in the standard Emden form (Chandrasekhar 1942)

$\frac{1}{\xi^{2}} \frac{\mathrm{d}}{\mathrm{d} \xi}\left(\xi^{2} \frac{\mathrm{d} \psi}{\mathrm{d} \xi}\right)=\mathrm{e}^{-\psi}$,

with $\psi=\psi^{\prime}=0$ at $\xi=0$. This equation has to be solved between $\xi=0$ and $\xi=\alpha$ corresponding to the normalized box radius

$\alpha=\left(4 \pi G \beta \rho_{0}\right)^{1 / 2} R$.

We also recall the definition of the Milne variables that will be used in the sequel

$u=\frac{\xi \mathrm{e}^{-\psi}}{\psi^{\prime}}, \quad v=\xi \psi^{\prime}$.

These variables satisfy the identities

$\frac{1}{u} \frac{\mathrm{d} u}{\mathrm{~d} \xi}=\frac{1}{\xi}(3-v-u)$,

$\frac{1}{v} \frac{\mathrm{d} v}{\mathrm{~d} \xi}=\frac{1}{\xi}(u-1)$,

which can be directly derived from Eq. (14).

The phase portrait of classical isothermal spheres is represented in Fig. 1 and forms a spiral. The spiral starts at $(u, v)=$ $(3,0)$ for $\xi=0$ and tends to the limit point $\left(u_{\mathrm{s}}, v_{\mathrm{s}}\right)=(1,2)$ as $\xi \rightarrow+\infty$. This limit point corresponds to the singular solution $\mathrm{e}^{-\psi_{\mathrm{s}}}=2 / \xi^{2}$. For finite isothermal spheres, we must consider only the part of the curve between $\xi=0$ and $\xi=\alpha$.

\subsection{The equilibrium phase diagram}

The thermodynamical parameters can be expressed in terms of the values of the Milne variables at the normalized box radius $\alpha$. Writing $u_{0}=u(\alpha)$ and $v_{0}=v(\alpha)$, the energy and the temperature can be written as (Padmanabhan 1989; Chavanis 2002a):

$\Lambda \equiv-\frac{E R}{G M^{2}}=\frac{1}{v_{0}}\left(\frac{3}{2}-u_{0}\right)$,

$\eta \equiv \frac{\beta G M}{R}=v_{0}$ 
We need also to express $\alpha$ in terms of the fugacity $z$. Using the expression of the gravitational potential at the box radius $\Phi(R)=-G M / R$ and comparing Eqs. (7) and (13), we get

$\left(\frac{2 \pi}{\beta}\right)^{3 / 2} z \mathrm{e}^{\eta}=\rho_{0} \mathrm{e}^{-\psi(\alpha)}$,

Expressing $\rho_{0}$ in terms of $\alpha$ by Eq. (15) and introducing the Milne variables (16), we obtain

$\chi \equiv \frac{4(2 \pi)^{5} G^{2} R^{4} z^{2}}{\beta}=u_{0}^{2} v_{0}^{2} \mathrm{e}^{-2 v_{0}}$.

We can also normalize the fugacity by the energy instead of the temperature. Combining Eqs. (22), (20) and (19), we get

$v \equiv-16(2 \pi){ }^{10} G^{5} R^{7} z^{4} E=u_{0}^{4} v_{0}^{5} \mathrm{e}^{-4 v_{0}}\left(\frac{3}{2}-u_{0}\right)$.

It is easy to show that statistical equilibrium states only exist for sufficiently small values of the control parameters $\Lambda, \eta, \chi$ and $v$ defined previously. Above a critical value, there is no possible equilibrium state and the system is expected to collapse. For example, for $\Lambda \geq \Lambda_{\mathrm{c}}=0.335$ (which corresponds to sufficiently negative energies), we have the well-know gravothermal catastrophe (Antonov 1962; Lynden-Bell \& Wood 1968). Similarly, for $\eta \geq \eta_{\mathrm{c}}=2.52$ (which corresponds to small temperatures or large masses), we have an isothermal collapse (Chavanis 2002a; Chavanis et al. 2002). We can show the existence of such bounds by a simple graphical construction, without numerical work. Indeed, the equilibrium structure of the system (characterized by its degree of central concentration $\alpha$ ) is determined by the intersection between the spiral in the $(u, v)$ plane and the curves defined by Eqs. (19), (20) and (22). If there is no intersection, the system cannot be in statistical equilibrium. This graphical construction is made explicitly in Fig. 1 in MCE, $\mathrm{CE}$ and GCE.

In the microcanonical ensemble, the control parameters are the energy $E$ and the particle number $N$. The parameter conjugate to the energy (with respect to the entropy $S$ ) is the inverse temperature $\beta$. The curve giving the inverse temperature as a function of energy for a fixed particle number $N$ is drawn in Fig. 2. The parameter conjugate to the particle number $N$ is the ratio $-\mu / T$, related to the fugacity $z$. In order to represent the fugacity as a function of $N$, for a fixed energy, we use Eqs. (19) and (23). The $-\mu / T$ vs. $N$ curve can then be deduced from Figs. 3 and 4. According to standard turning point arguments (Katz 1978), the series of equilibria becomes unstable at the point of minimum energy (for a given mass) or at the point of minimum mass (for a given negative energy). The condition $d \Lambda / d \alpha=0$ implies (Padmanabhan 1989)

$4 u_{0}^{2}+2 u_{0} v_{0}-11 u_{0}+3=0$.

The values of $\alpha$ at which the parameter $\Lambda$ is extremum are determined by the intersections between the spiral in the $(u, v)$ plane and the parabole defined by Eq. (24), see Fig. 5. The change of stability occurs for the smallest value of $\alpha$. Its numerical value is $\alpha=34.4$ corresponding to a density contrast of 709. Gravitational instability occurs in MCE when the specific

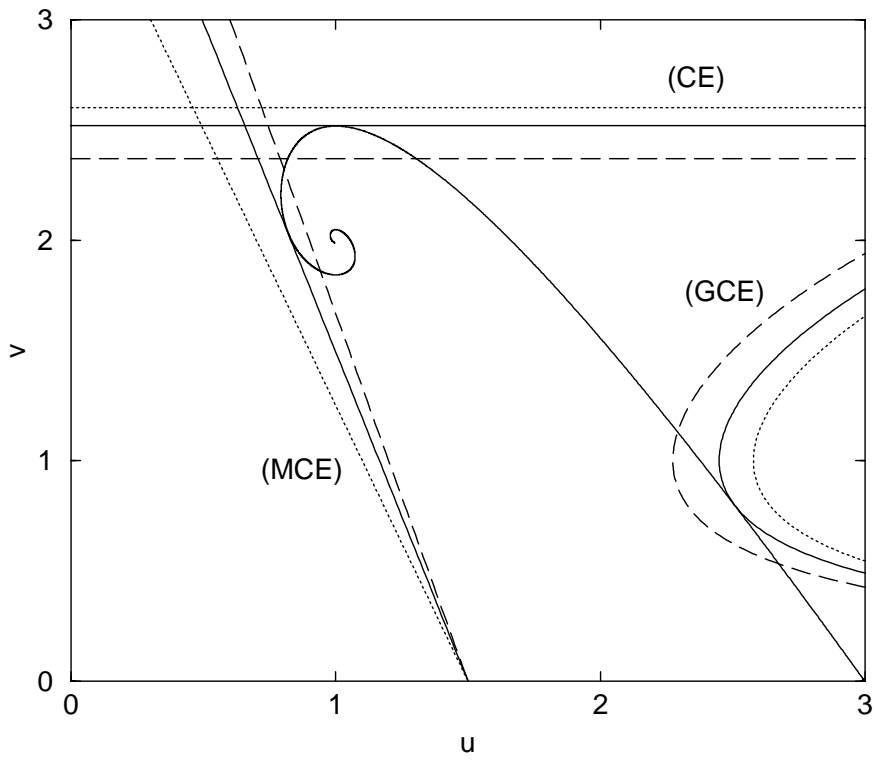

Fig. 1. This graphical construction, first introduced by Padmanabhan (1989) in MCE, shows very simply the existence of an upper bound for $\Lambda, \eta$ and $\chi$ above which there is no hydrostatic equilibrium for an isothermal gas (dotted line: $\Lambda>\Lambda_{c}$; solid line: $\Lambda=\Lambda_{c}$; dashed line: $\Lambda<\Lambda_{\mathrm{c}}$ with similar convention for $\eta$ and $\chi$ ).

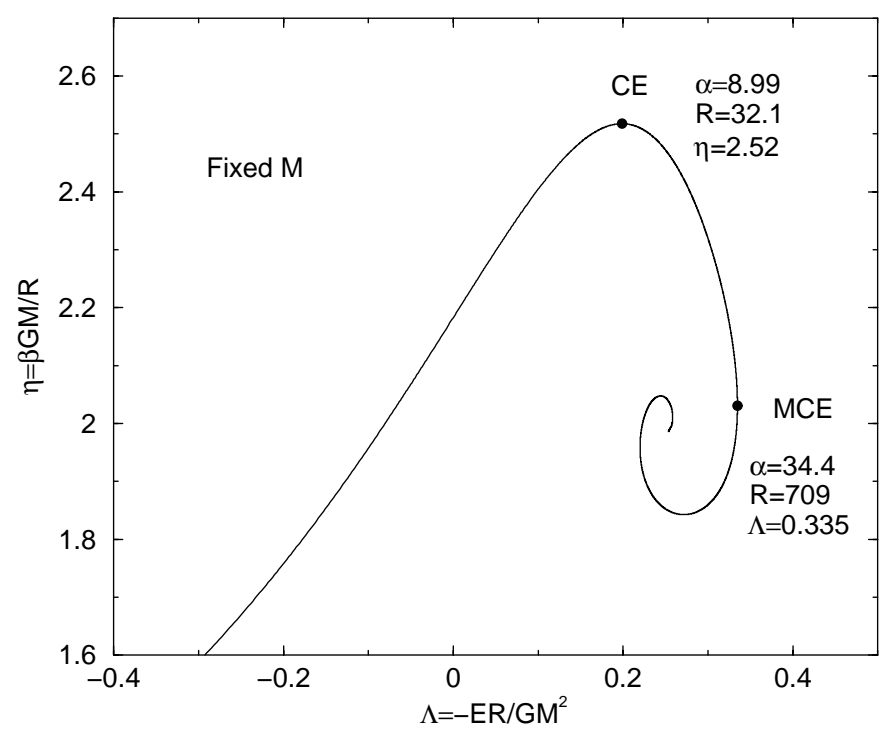

Fig. 2. Temperature vs. energy plot at fixed particle number. The series of equilibria becomes unstable at MCE in the microcanonical ensemble and at $\mathrm{CE}$ in the canonical ensemble.

heats $C_{V}=(\partial E / \partial T)_{N, V}$ becomes zero, passing from negative to positive values.

In the canonical ensemble, the control parameters are the inverse temperature $\beta$ and the particle number $N$. The parameter conjugate to the inverse temperature (with respect to the free energy $J$ ) is $-E$. The curve giving $-E$ as a function of $\beta$ for a fixed particle number can be deduced from Fig. 2. The parameter conjugate to the particle number is $-\mu / T$, related to the fugacity $z$. We can represent the fugacity as a function of mass, for a given temperature, by using the relations (22) and (20). The corresponding curve can be deduced from the $\eta$ vs. $\chi$ plot represented in Fig. 6. In the canonical ensemble, the series of 


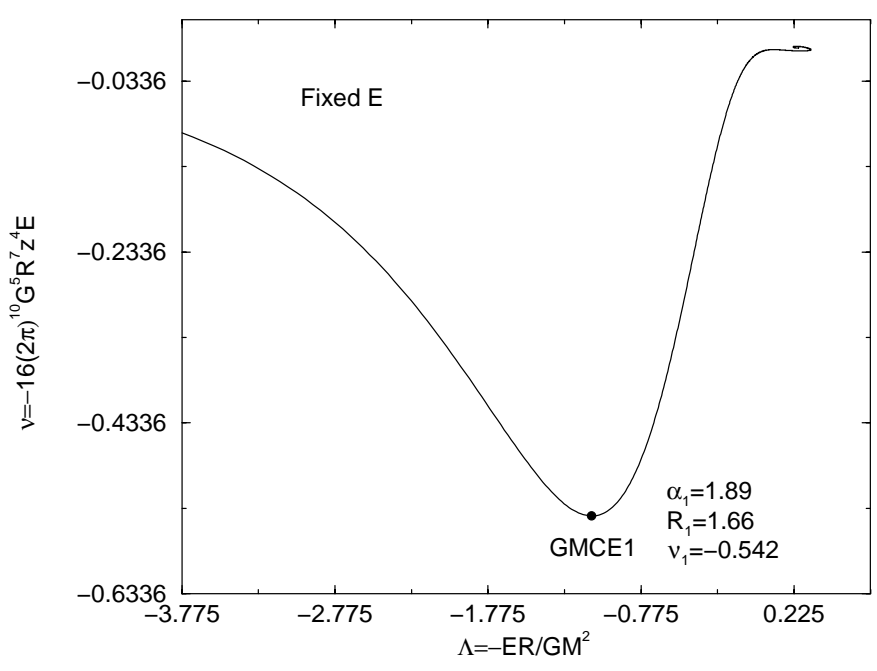

Fig. 3. Fugacity vs. particle number at fixed energy. The series of equilibria becomes unstable at GMCE1 in the grand microcanonical ensemble.

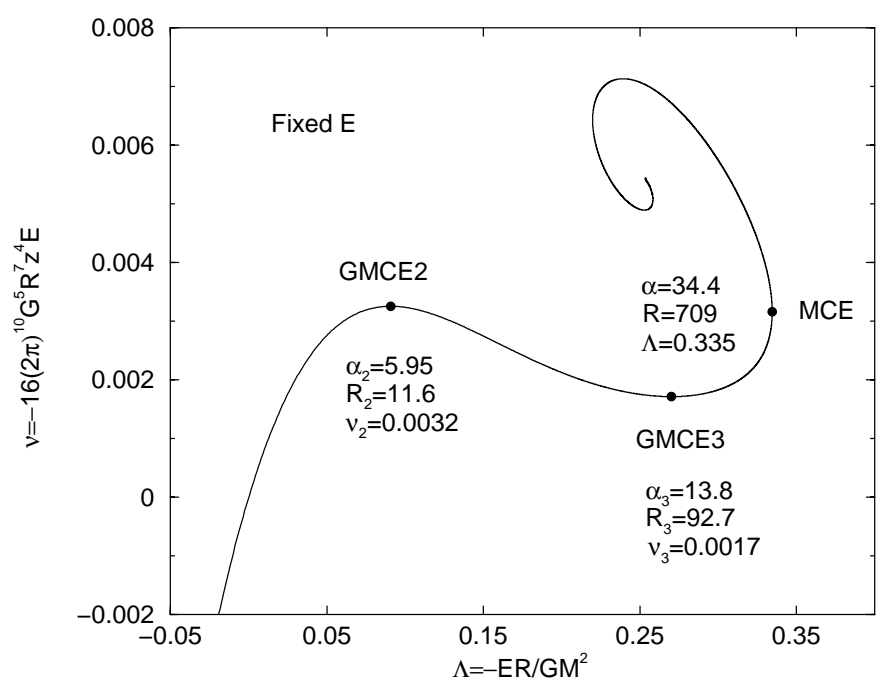

Fig. 4. Enlargement of Fig. 3 near the spiral. In the grand microcanonical ensemble, a change of stability occurs at GMCE2 and GMCE3. Between these points the series of equilibria is stable again. After GMCE3, the series becomes and remains unstable.

equilibria becomes unstable at the point of minimum temperature (for a given mass) or at the point of maximum mass (for a given temperature). The condition $\mathrm{d} \eta / \mathrm{d} \alpha=0$ is equivalent to (Chavanis 2002a)

$u_{0}=1$.

The values of $\alpha$ at which the parameter $\eta$ is extremum are determined by the intersections between the spiral in the $(u, v)$ plane and the straight line defined by Eq. (25), see Fig. 5. The change of stability occurs for the smallest value of $\alpha$. Its numerical value is $\alpha=8.99$ corresponding to a density contrast of 32.1. Gravitational instability in CE occurs when the specific heats becomes negative passing by $C_{V}=\infty$.

In the grand canonical ensemble, the control parameters are the inverse temperature $\beta$ and the ratio $\mu / T$ related to the fugacity $z$. The parameter conjugate to the inverse temperature (with

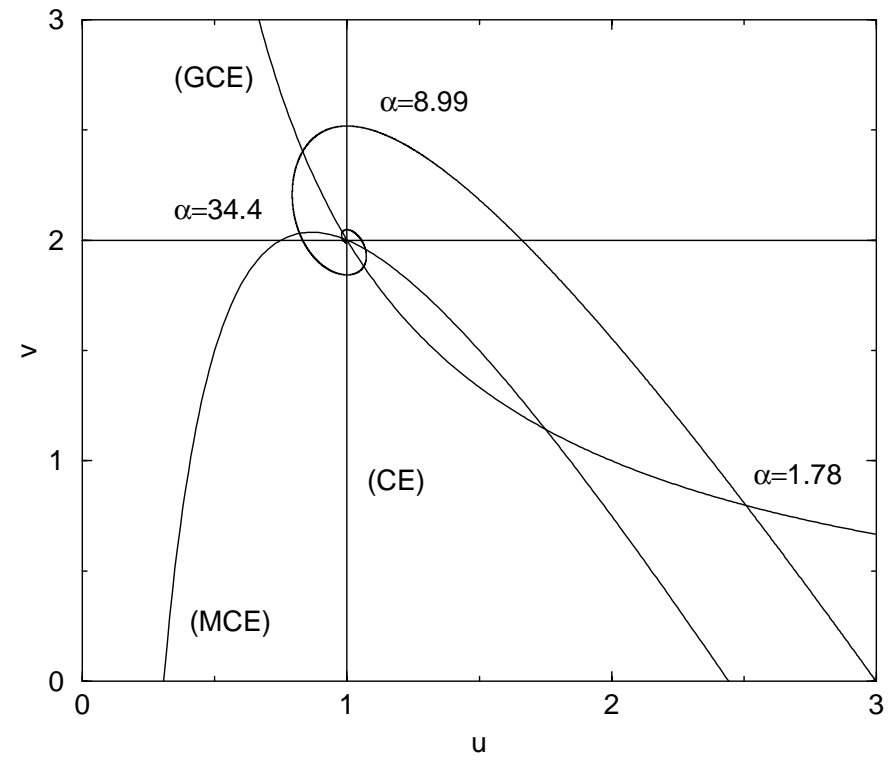

Fig. 5. Graphical construction determining the turning points of the control parameter at which a change of stability occurs in MCE, CE and GCE.

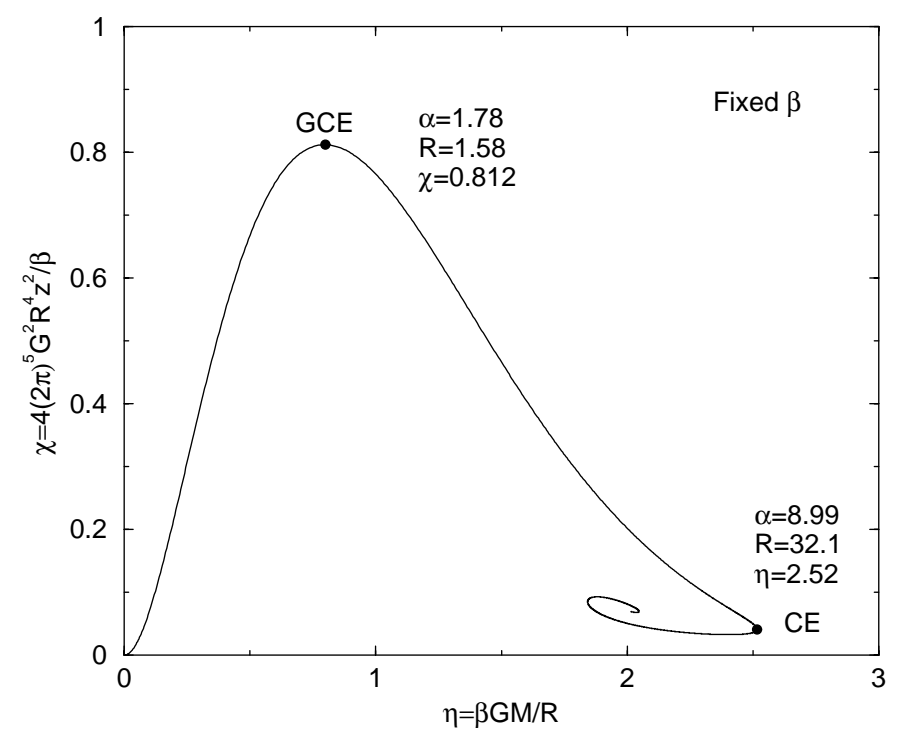

Fig. 6. Fugacity vs. mass at fixed temperature. The series of equilibria becomes unstable at GCE in the grand canonical ensemble.

respect to the grand potential $G$ ) is $-E$. The curve giving $-E$ as a function of $\beta$ for a fixed fugacity $z$ is determined by Eqs. (22) and (23) and can be deduced from Figs. 7 and 8. The parameter conjugate to the ratio $\mu / T$ is the particle number $N$. The curve giving $N$ as a function of $z$ for a given temperature can be deduced from Fig. 6. In the grand canonical ensemble, the series of equilibria becomes unstable at the point of maximum temperature (for a given fugacity) or at the point of maximum fugacity (for a given temperature). The condition $\mathrm{d} \chi / \mathrm{d} \alpha=0$ implies

$u_{0} v_{0}=2$.

The values of $\alpha$ at which the parameter $\chi$ is extremum (see Fig. 9) are determined by the intersections between the spiral 


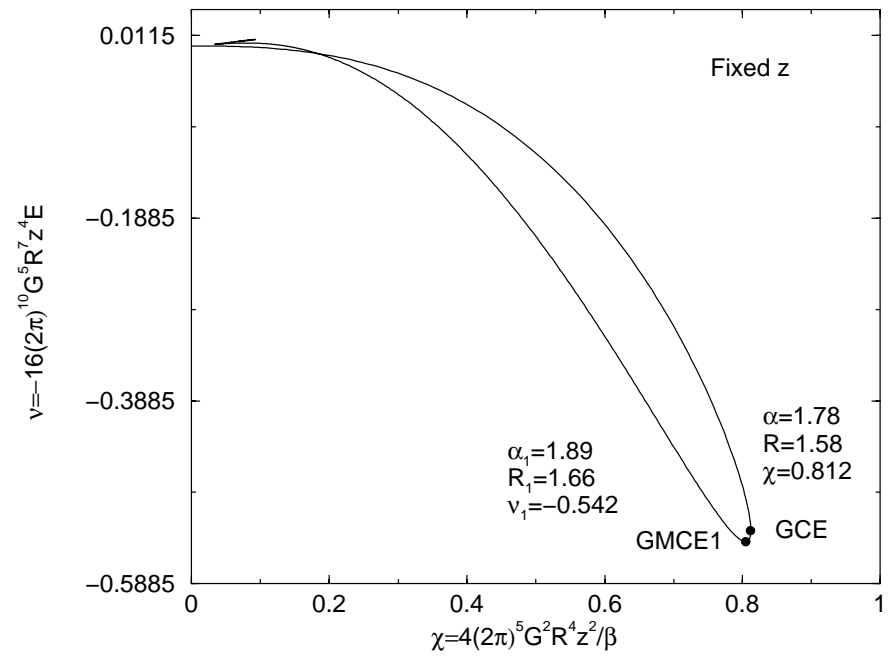

Fig. 7. Energy vs. temperature at fixed fugacity.

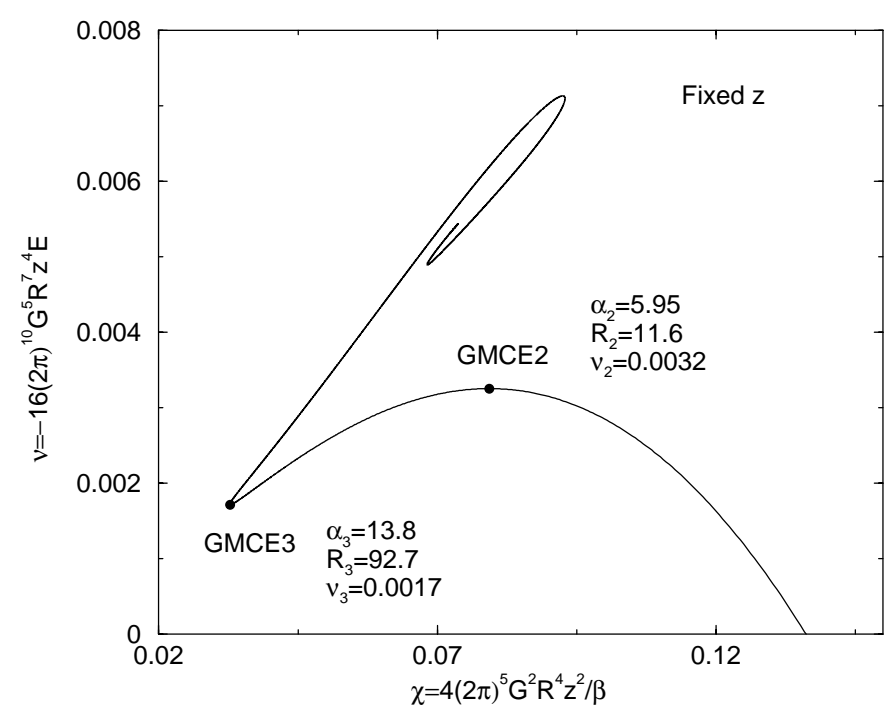

Fig. 8. Enlargement of Fig. 7 near the spiral.

in the $(u, v)$ plane and the hyperbole defined by Eq. (26), see Fig. 5. The change of stability occurs for the smallest value of $\alpha$. Its numerical value is $\alpha=1.78$ corresponding to a density contrast of 1.58 .

In the grand microcanonical ensemble, the control parameters are the energy $E$ and the ratio $\mu / T$ related to the fugacity $z$. The parameter conjugate to the energy (with respect to the potential $\mathcal{K}$ ) is $\beta$. The curve giving $\beta$ as a function of $E$ for a fixed fugacity $z$ can be deduced from Figs. 7 and 8 . The parameter conjugate to the ratio $\mu / T$ is the particle number $N$. The $N$ vs. $\mu / T$ plot for a given energy can be deduced from Figs. 3 and 4. In the grand microcanonical ensemble, the series of equilibria becomes unstable at the point of maximum energy (for a given fugacity) or at the point of maximum fugacity (for a given positive energy). The condition $\mathrm{d} v / \mathrm{d} \alpha=0$ implies

$8 u_{0}^{2} v_{0}-10 u_{0} v_{0}-17 u_{0}+21=0$.

The values of $\alpha$ at which the parameter $v$ is extremum (see Fig. 10) are determined by the intersections between the spiral in the $(u, v)$ plane and the curve defined by Eq. (27), see Fig. 11.

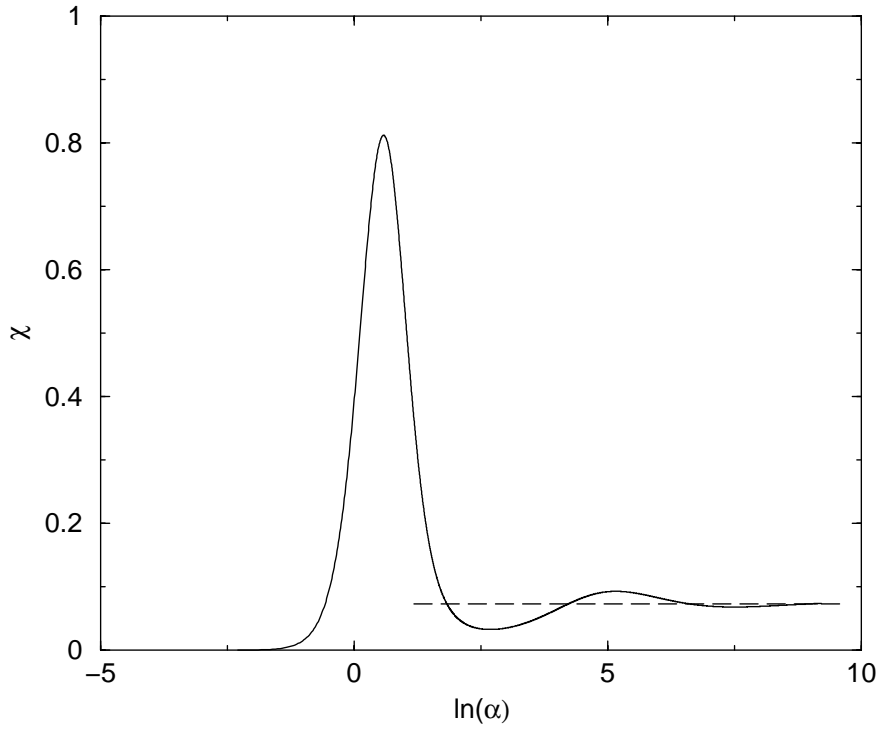

Fig. 9. Evolution of $\chi$ as a function of the central concentration $\alpha$. In dashed line we have represented the asymptotic value $\chi_{\mathrm{s}}=4 \mathrm{e}^{-4}$ corresponding to the singular solution. The $\Lambda(\alpha)$ and $\eta(\alpha)$ curves have a similar behaviour.

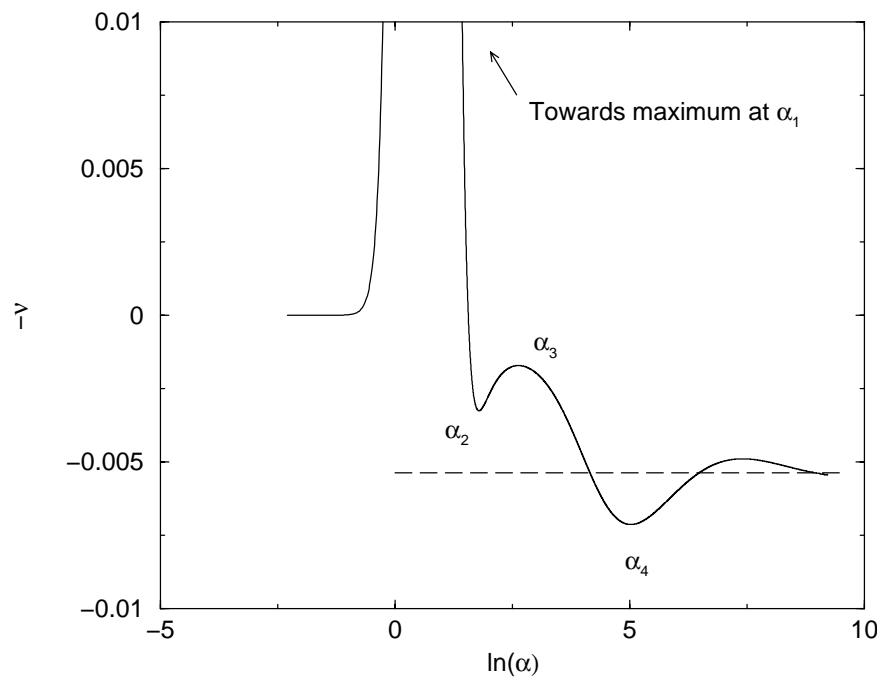

Fig. 10. Evolution of $v$ as a function of the central concentration $\alpha$. In dashed line we have represented the asymptotic value $v_{\mathrm{s}}=2^{4} \mathrm{e}^{-8}$ corresponding to the singular solution. Contrary to Fig. 9, the second extremum $\alpha_{2}$ is above the asymptote $v=v_{\mathrm{s}}$. This appears to be the reason for the regain of stability between $\alpha_{2}$ and $\alpha_{3}$.

The series of equilibria becomes unstable for $\alpha_{1}=1.89$ (density contrast 1.66) which corresponds to the first intersection. At that point, the $v$ vs. $\chi$ curve rotates clockwise (see Fig. 7). However, at the second turning point $\alpha_{2}=5.95$ (density contrast 11.6) the $v$ vs. $\chi$ curve rotates anticlockwise (see Fig. 8) so that stability is regained (this is proved in Katz 1978). Stability is lost again at $\alpha_{3}=13.8$ (density contrast 92.7) and never recovered afterwards. This curious behaviour in GMCE was first noted by Lecar \& Katz (1981). A similar lost-gain-lost stability behaviour also occurs for self-gravitating fermions and hard sphere models in MCE and CE (Chavanis \& Sommeria 1998; 


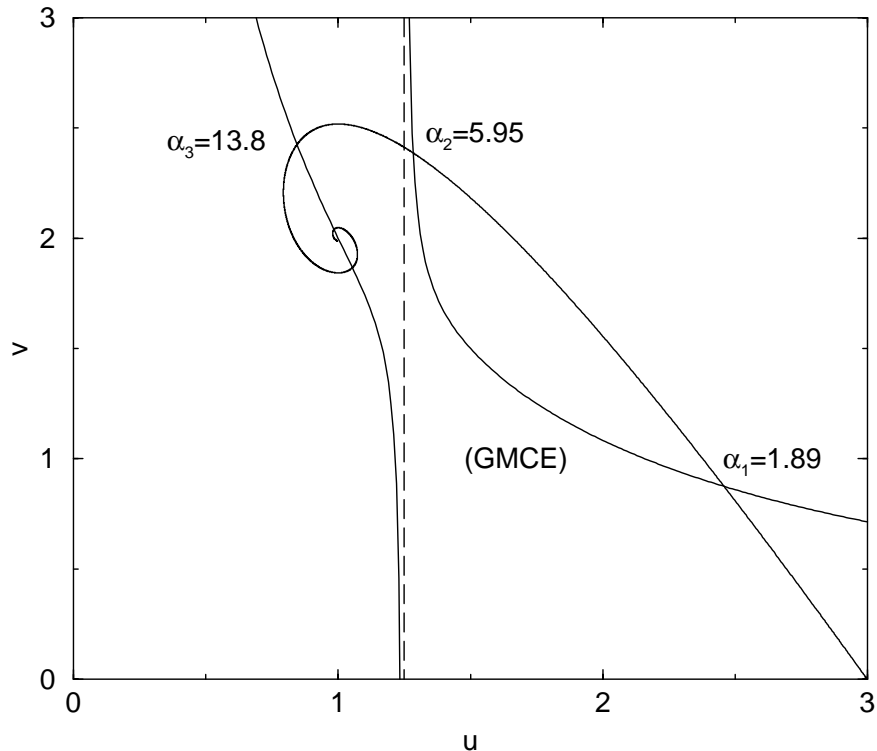

Fig. 11. Graphical construction determining the turning points of the control parameter $v$ in GMCE. The series of equilibria is stable for $\alpha \leq \alpha_{1}$, unstable for $\alpha_{1} \leq \alpha \leq \alpha_{2}$, stable again for $\alpha_{2} \leq \alpha \leq \alpha_{3}$ and unstable for $\alpha \geq \alpha_{3}$.

Chavanis 2002c). In that case, it is related to the occurence of phase transitions between "gaseous" and "condensed" states.

\section{Thermodynamical stability of self-gravitating systems}

We now determine the condition of thermodynamical stability by computing the second order variations of the thermodynamical potential explicitly. This study has already been done in MCE (Padmanabhan 1989) and CE (Chavanis 2002a). In the following, we perform the analysis in GCE and GMCE.

\subsection{The grand canonical ensemble}

The second order variations of the grand potential $G$ are

$\delta^{2} G=-\frac{k}{m} \int \frac{(\delta \rho)^{2}}{2 \rho} \mathrm{d}^{3} \boldsymbol{r}-\frac{1}{2 T} \int \delta \rho \delta \Phi \mathrm{d}^{3} \boldsymbol{r}$.

An isothermal sphere is stable in the grand canonical ensemble if $\delta^{2} G \leq 0$ for all variations $\delta \rho$ of the density profile. We shall restrict ourselves to spherically symmetric perturbations since only such perturbations can trigger gravitational instability for non rotating systems (Horwitz \& Katz 1978). In the grand canonical ensemble, the mass of the system is not conserved. Therefore, the potential at the wall changes according to

$\delta \Phi(R)=-\frac{G \delta M}{R}$.

On the other hand, according to the Gauss theorem, we have

$\frac{\mathrm{d} \delta \Phi}{\mathrm{d} r}(R)=\frac{G \delta M}{R^{2}}$
These boundary conditions suggest the introduction of the new function

$X(r)=r \delta \Phi(r)$,

which satisfies

$X(0)=0, \quad X^{\prime \prime}(0)=0, \quad X^{\prime}(R)=0$,

where we have used Eqs. (29) and (30) to obtain the third equality (the second equality is obvious for a spherically symmetric system).

The variations $\delta \Phi$ and $\delta \rho$ are related to each other by the Poisson equation

$\frac{1}{r^{2}} \frac{\mathrm{d}}{\mathrm{d} r}\left(r^{2} \frac{\mathrm{d} \delta \Phi}{\mathrm{d} r}\right)=4 \pi G \delta \rho$.

In terms of the new variable (31), Eq. (33) becomes

$\frac{X^{\prime \prime}}{r}=4 \pi G \delta \rho$.

Expressing the second order variations of $G$ in terms of $X$, we get

$\delta^{2} G=-\frac{k}{m} \frac{1}{8 \pi G^{2}} \int_{0}^{R} \frac{\left(X^{\prime \prime}\right)^{2}}{\rho} \mathrm{d} r-\frac{1}{2 T G} \int_{0}^{R} X^{\prime \prime} X \mathrm{~d} r$.

Integrating by parts, we can put Eq. (35) in the form

$\delta^{2} G=\frac{1}{2 G^{2}} \int_{0}^{R} \mathrm{~d} r X^{\prime}\left[\frac{G}{T}+\frac{k}{m} \frac{\mathrm{d}}{\mathrm{d} r}\left(\frac{1}{4 \pi \rho} \frac{\mathrm{d}}{\mathrm{d} r}\right)\right] X^{\prime}$.

The boundary terms arising from the integration by parts are seen to vanish by virtue of Eq. (32). We can therefore reduce the stability analysis of isothermal spheres in the grand canonical ensemble to the eigenvalue problem

$\left[\frac{k}{m} \frac{\mathrm{d}}{\mathrm{d} r}\left(\frac{1}{4 \pi \rho} \frac{\mathrm{d}}{\mathrm{d} r}\right)+\frac{G}{T}\right] X_{\lambda}^{\prime}(r)=\lambda X_{\lambda}^{\prime}(r)$.

If all the eigenvalues $\lambda$ are negative, then the isothermal sphere is a maximum of the thermodynamical potential $G$ (and is therefore stable in GCE). If at least one eigenvalue is positive, it is an unstable saddle point. The condition of marginal stability $\lambda=0$ determines the transition between stability and instability. We thus have to solve the equation

$\frac{k}{m} \frac{\mathrm{d}}{\mathrm{d} r}\left(\frac{1}{4 \pi \rho} \frac{\mathrm{d} X^{\prime}}{\mathrm{d} r}\right)+\frac{G X^{\prime}}{T}=0$.

Integrating once and using the boundary conditions (32) at $r=0$, the constant of integration is seen to vanish and we get

$\frac{k}{m} \frac{1}{4 \pi \rho} X^{\prime \prime}+\frac{G X}{T}=0$.

Introducing the dimensionless variables defined in Sect. 2.3, we are led to solve the problem

$\mathrm{e}^{\psi} X^{\prime \prime}+X=0$

$X(0)=X^{\prime}(\alpha)=0$.

Let us denote by

$\mathcal{L} \equiv \mathrm{e}^{\psi} \frac{\mathrm{d}^{2}}{\mathrm{~d} \xi^{2}}+1$, 
the differential operator which occurs in Eq. (40). Using the Emden Eq. (14), we readily check that

$\mathcal{L}(\xi)=\xi, \quad \mathcal{L}\left(\xi^{2} \psi^{\prime}\right)=2 \xi, \quad \mathcal{L}(\xi \psi)=\xi+\xi \psi$.

Therefore, the general solution of Eq. (40) which satisfies the condition $X=0$ at the origin is

$X(\xi)=c_{1} \xi\left(\xi \psi^{\prime}-2\right)$,

where $c_{1}$ is an arbitrary constant. The boundary condition $X^{\prime}(\alpha)=0$ implies

$\alpha^{2} \mathrm{e}^{-\psi(\alpha)}-2=0$.

This relation determines the value of $\alpha$ at which the series of equilibria becomes unstable in the grand canonical ensemble. In terms of the Milne variables, Eq. (45) is equivalent to

$u_{0} v_{0}=2$,

which is precisely the condition (26) obtained with the turning point criterion. We are now in a position to determine the structure of the perturbation profile that triggers the instability in GCE. Using Eq. (34), we have

$\frac{\delta \rho}{\rho_{0}}=\frac{X^{\prime \prime}}{4 \pi \xi}$

with Eq. (44) for $X$. This yields

$\frac{\delta \rho}{\rho_{0}}=\frac{c_{1}}{4 \pi} \mathrm{e}^{-\psi}\left(2-\xi \psi^{\prime}\right)$,

or, introducing the Milne variables,

$\frac{\delta \rho}{\rho}=\frac{c_{1}}{4 \pi}(2-v)$.

We note that Eq. (49) coincides with the expression found in the canonical ensemble (Chavanis 2002a). The number of nodes in the profile $\delta \rho$ is determined by the number of intersections between the spiral in the $(u, v)$ plane and the line $v=2$. We see in Fig. 5 that there is no intersection for $\xi \leq \alpha=1.78$, where 1.78 is the stability limit in GCE. Therefore, collecting the results obtained by different authors, the perturbation that triggers gravitational instability has two nodes in MCE ("core-halo" structure), one node in CE and no node in GCE (see Fig. 12).

\subsection{The grand microcanonical ensemble}

The grand microcanonical ensemble is interesting in its own right but also because it exhibits a lost-gain-lost stability behaviour similar to that found for self-gravitating fermions and hard sphere models (Chavanis 2002c). Now, the stability analysis can be conducted analytically in GMCE for classical isothermal spheres while only numerical results are available for fermions and hard sphere systems. On a technical point of view, GMCE is the most complicated and richest of all thermodynamical ensembles. We can argue, however, that GMCE may not be realized in practice. It is not clear, indeed, what mechanism can be devised so that two systems can share particles without also sharing energy. One example might be the case of

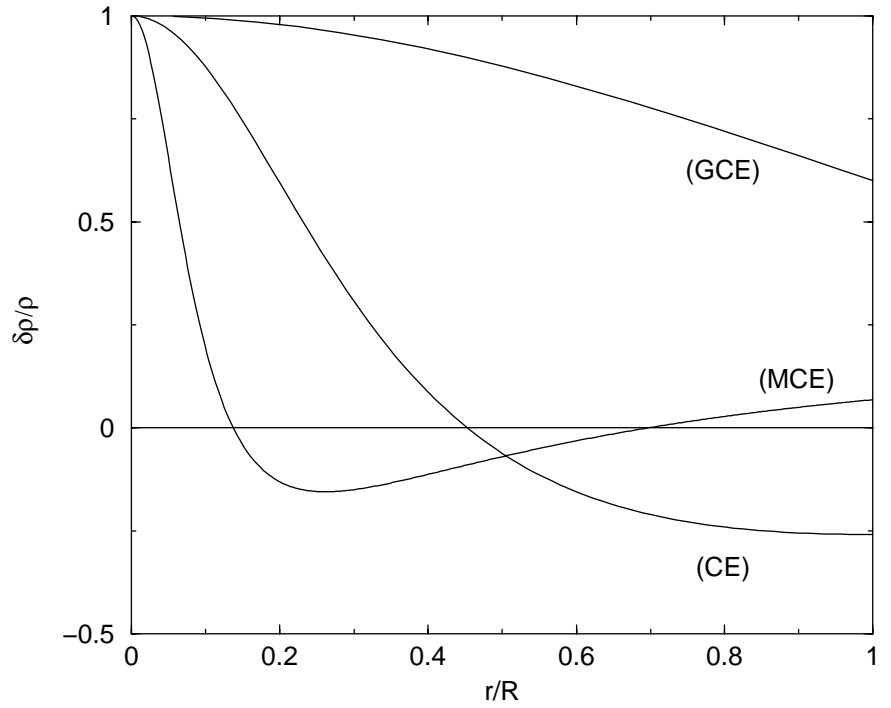

Fig. 12. First mode of instability in MCE, CE and GCE.

relativistic particles created and destroyed at equilibrium in a cosmological settling.

In GMCE, we have to maximize the thermodynamical potential

$$
\begin{aligned}
\frac{\mathcal{K}}{k}= & {\left[\frac{5}{2}+\frac{3}{2} \ln \left(\frac{2 \pi k T}{m}\right)+\frac{\mu}{k T}\right] \int \frac{\rho}{m} \mathrm{~d}^{3} \boldsymbol{r} } \\
& -\int \frac{\rho}{m} \ln \frac{\rho}{m} \mathrm{~d}^{3} \boldsymbol{r},
\end{aligned}
$$

at fixed energy

$E=\frac{3}{2} k T \int \frac{\rho}{m} \mathrm{~d}^{3} \boldsymbol{r}+\frac{1}{2} \int \rho \Phi \mathrm{d}^{3} \boldsymbol{r}$.

We vary the density profile and use Eq. (51) to determine the corresponding variation of temperature. After some algebra, we find that the second order variations of the thermodynamical potential can be written

$$
\begin{aligned}
\delta^{2} \mathcal{K}= & -\frac{1}{2 T} \int \delta \rho \delta \Phi \mathrm{d}^{3} \boldsymbol{r}-\frac{k}{m} \int \frac{(\delta \rho)^{2}}{2 \rho} \mathrm{d}^{3} \boldsymbol{r} \\
& -\frac{1}{3 N k T^{2}}\left(\int\left(\Phi+\frac{3 k T}{2 m}\right) \delta \rho \mathrm{d}^{3} \boldsymbol{r}\right)^{2}
\end{aligned}
$$

Introducing the variable $X$ defined previously and integrating by parts, we can put the problem in the form

$\delta^{2} \mathcal{K}=\frac{1}{2 G^{2}} \int_{0}^{R} \mathrm{~d} r \int_{0}^{R} \mathrm{~d} r^{\prime} X^{\prime}(r) K\left(r, r^{\prime}\right) X^{\prime}\left(r^{\prime}\right)$,

with

$$
\begin{aligned}
K\left(r, r^{\prime}\right)= & \left\{\frac{G}{T}+\frac{k}{m} \frac{\mathrm{d}}{\mathrm{d} r}\left(\frac{1}{4 \pi \rho} \frac{\mathrm{d}}{\mathrm{d} r}\right)\right\} \delta\left(r-r^{\prime}\right) \\
& -\frac{2}{3 N k T^{2}}\left[\left(\Phi+\frac{3 k T}{2 m}\right) r\right]^{\prime}(r)\left[\left(\Phi+\frac{3 k T}{2 m}\right) r\right]^{\prime}\left(r^{\prime}\right)
\end{aligned}
$$

The stability analysis reduces therefore to the study of the eigenvalue equation

$\int_{0}^{R} \mathrm{~d} r^{\prime} K\left(r, r^{\prime}\right) X_{\lambda}^{\prime}\left(r^{\prime}\right)=\lambda X_{\lambda}^{\prime}(r)$. 
The condition of marginal stability $(\lambda=0)$ reads

$$
\begin{aligned}
\frac{G X^{\prime}}{T}+\frac{k}{m} \frac{\mathrm{d}}{\mathrm{d} r}\left(\frac{1}{4 \pi \rho} \frac{\mathrm{d} X^{\prime}}{\mathrm{d} r}\right)= & \frac{2}{3 N k T^{2}}\left[\left(\Phi+\frac{3 k T}{2 m}\right) r\right]^{\prime} \\
& \times \int_{0}^{R}\left[\left(\Phi+\frac{3 k T}{2 m}\right) r\right]^{\prime} X^{\prime} \mathrm{d} r .
\end{aligned}
$$

Integrating once, we obtain

$$
\begin{aligned}
\frac{G X}{T}+\frac{k}{m} \frac{X^{\prime \prime}}{4 \pi \rho}= & \frac{2}{3 N k T^{2}}\left(\Phi+\frac{3 k T}{2 m}\right) r \\
& \times \int_{0}^{R}\left[\left(\Phi+\frac{3 k T}{2 m}\right) r\right]^{\prime} X^{\prime} \mathrm{d} r .
\end{aligned}
$$

The gravitational potential $\Phi$ is related to the Emden function $\psi(\xi)$ by the relation $\psi=\beta\left(\Phi-\Phi_{0}\right)$. Using $\Phi(R)=-G M / R$ and Eq. (20), we get

$\beta \Phi=\psi-\psi(\alpha)-v_{0}$

Inserting this relation in Eq. (57) and introducing the dimensionless variables defined in Sect. 2.3, we find that

$$
\begin{aligned}
X+\mathrm{e}^{\psi} X^{\prime \prime}= & \frac{1}{\alpha v_{0}}\left[\psi-\psi(\alpha)-v_{0}+\frac{3}{2}\right] \xi \\
& \times\left\{-\frac{2}{3} \int_{0}^{\alpha} X \xi \mathrm{e}^{-\psi} \mathrm{d} \xi+X(\alpha)\right\},
\end{aligned}
$$

where we have integrated by parts and used the identity $(\xi \psi)^{\prime \prime}=\xi \mathrm{e}^{-\psi}$ equivalent to the Emden Eq. (14). Writing

$V=\frac{2}{3 v_{0} \alpha}\left(\frac{3}{2} X(\alpha)-\int_{0}^{\alpha} X \xi \mathrm{e}^{-\psi} \mathrm{d} \xi\right)$,

$W=-\left[\psi(\alpha)+v_{0}-\frac{3}{2}\right] V$,

we are led to solve the problem

$\mathrm{e}^{\psi} X^{\prime \prime}+X=V \psi \xi+W \xi$

$X(0)=X^{\prime}(\alpha)=0$.

Seeking a solution of Eq. (62) in the form

$X=a_{1} \xi+a_{2} \xi^{2} \psi^{\prime}+a_{3} \xi \psi$,

and using the identities (43), we find that

$X=(W-V) \xi+b\left(\xi^{2} \psi^{\prime}-2 \xi\right)+V \xi \psi$.

The constant $b$ is determined by the boundary condition $X^{\prime}(\alpha)=0$. We thus find that the general solution of Eqs. (62) and (63) is

$$
\begin{aligned}
X(\xi)= & -\left[\psi(\alpha)+v_{0}-\frac{1}{2}\right] V \xi+V \xi \psi \\
& -\frac{V}{2} \frac{1}{u_{0} v_{0}-2}\left(\xi^{2} \psi^{\prime}-2 \xi\right),
\end{aligned}
$$

where we have used Eq. (61). The values of $\alpha$ at which a change of stability occurs $(\lambda=0)$ are obtained by inserting the solution (66) for $X$ in Eq. (60). We need the identities

$$
\begin{aligned}
& \int_{0}^{\alpha} \xi^{2} \mathrm{e}^{-\psi} \mathrm{d} \xi=\alpha v_{0} \\
& \int_{0}^{\alpha} \xi^{3} \psi^{\prime} \mathrm{e}^{-\psi} \mathrm{d} \xi=\alpha v_{0}\left(3-u_{0}\right), \\
& \int_{0}^{\alpha} \xi^{2} \psi \mathrm{e}^{-\psi} \mathrm{d} \xi=\alpha v_{0}\left(\psi(\alpha)+v_{0}-6+2 u_{0}\right) .
\end{aligned}
$$

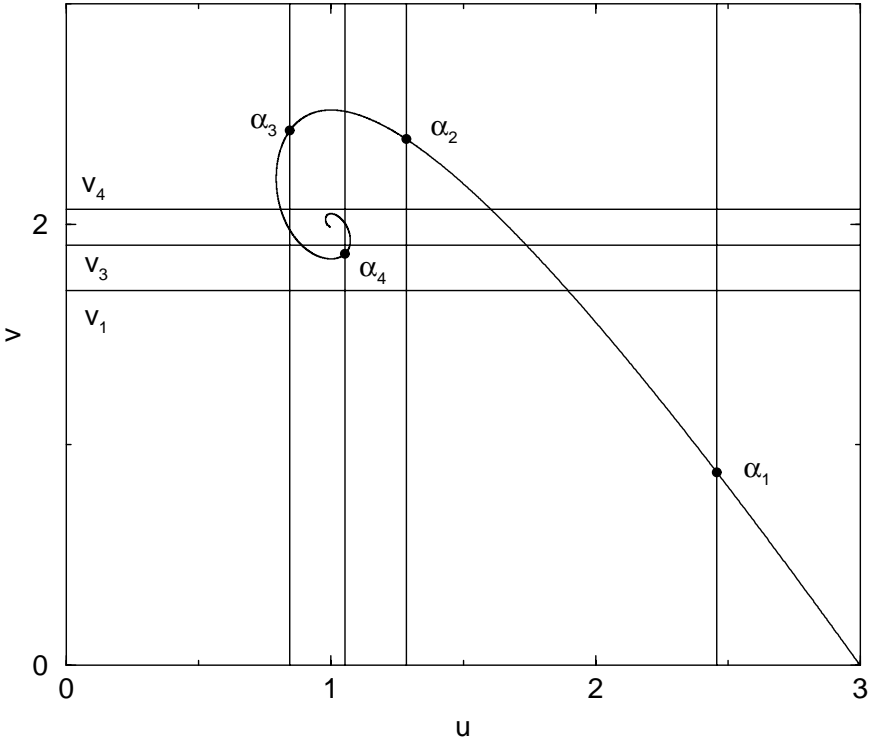

Fig. 13. Graphical construction determining the number of nodes for the different modes of instability in GMCE. The horizontal lines correspond to the condition (72) for the four first critical points.

which are derived in Appendix A. After simplification, we obtain the condition

$8 u_{0}^{2} v_{0}-10 u_{0} v_{0}-17 u_{0}+21=0$,

which coincides with the condition (27) deduced from the turning point criterion. The perturbation profile at the critical points at which $\lambda=0$ is given by Eq. (47) with Eq. (66) for $X$. Using Eqs. (13), (62) and (66), we get

$\frac{\delta \rho}{\rho}=\frac{c_{1}}{4 \pi}\left(2 u_{0} v_{0}-6+v\right)$,

where $c_{1}$ is a constant. The nodes of the perturbation profile are determined by the condition

$v\left(\xi_{i}\right)=6-2 u_{0} v_{0}$,

with $\xi_{i} \leq \alpha$. The number of nodes can be found by a graphical construction. In Fig. 13, we plot the lines defined by Eq. (72) in the $(u, v)$ plane for the four first critical points in the series of equilibria. The corresponding perturbation density profiles are plotted in Fig. 14. The first mode of instability $\alpha_{1}$ has no node. The profile of the second mode $\alpha_{2}$ increases (in absolute value) with distance. The absence of node at $\alpha_{2}$ is a signature of the fact that stability is regained at that point. The mode $\alpha_{3}$ at which stability is lost again has one node, the mode $\alpha_{4}$ two nodes etc.

\section{Connexion with statistical mechanics}

In this section, we briefly discuss the relation between the thermodynamics and the statistical mechanics (and field theory) of self-gravitating systems. We shall just discuss the physical ideas without entering into technical details.

In the microcanonical ensemble, the object of fundamental interest is the density of states

$g(E)=\int \delta(E-H) \prod_{i=1}^{N} \mathrm{~d}^{3} \boldsymbol{r}_{i} \mathrm{~d}^{3} \boldsymbol{v}_{i}$, 


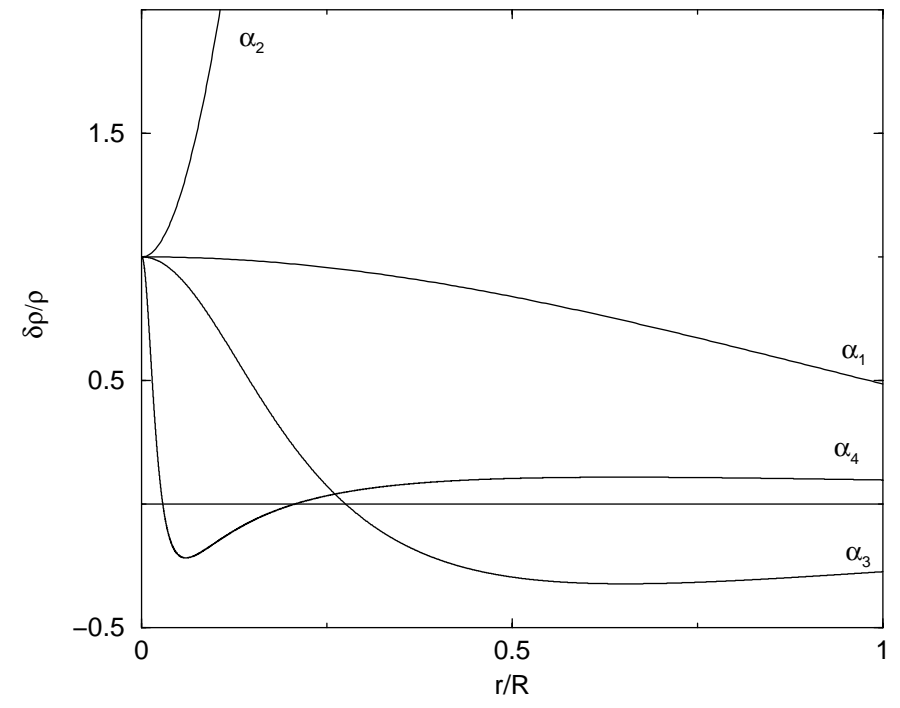

Fig. 14. Density perturbation profiles for the four first critical points in the series of equilibria in GMCE. We have fixed the constant $c_{1}$ such that $\delta \rho / \rho=1$ at $r=0$.

where

$H=\frac{1}{2} \sum_{i=1}^{N} m v_{i}^{2}-\sum_{i \leq j} \frac{G m^{2}}{\left|\boldsymbol{r}_{i}-\boldsymbol{r}_{j}\right|}$,

is the Hamiltonian of the self-gravitating gas. By definition, $g(E) \mathrm{d} E$ is proportional to the number of microstates (specified by the position and the velocity of all $N$ particles) with energy between $E$ and $E+\mathrm{d} E$. The entropy is defined by

$S(E)=\ln g(E)$.

As is customary in statistical mechanics, we decompose phase space into macrocells and divide these macrocells into a large number of microcells. A macrostate is characterized by the number of particles in each macrocell (irrespective of their precise position and velocity in the cell), or equivalently by the smooth distribution function $f(\boldsymbol{r}, \boldsymbol{v})$. If we call $W_{i}$ the number of microstates corresponding to the macrostate $i$, we can rewrite Eq. (73) formally in the form

$g(E)=\sum_{E_{i}=E} W_{i}=\sum_{E_{i}=E} \mathrm{e}^{S_{i}}=\sum_{E_{i}=E} \mathrm{e}^{N s_{i}}$,

where the sum runs over all macrostates with energy $E$ (and mass $M$ ). In addition, $S_{i}=\ln W_{i}$ is the Boltzmann entropy (4) of the macrostate $i$ (i.e., $S_{i} \equiv S[f]$ ) and $s_{i}=S_{i} / N$ the entropy per particle. The thermodynamic limit for self-gravitating systems corresponds to $N \rightarrow+\infty$ in such a way that all the dimensionless control parameters defined in Sect. 2.4 remain finite. This can be written for example $N, R \rightarrow+\infty$ with $N / R$ finite (and $E / N \sim 1, \beta \sim 1$ ). This is a very unusual thermodynamic limit due to the long-range nature of the interactions. Now, at the thermodynamic limit, the sum in Eq. (76) is dominated by the macrostate $f_{*}$ which maximizes the Boltzmann entropy (4) at fixed mass and energy:

$g(E) \simeq \mathrm{e}^{S\left[f_{*}\right]}, \quad S(E)=S\left[f_{*}\right]$.
It has to be noted that the sum in Eq. (76) is sharply peaked around $f_{*}$, which corresponds to what is called a large deviation property in mathematics. Therefore, the mean-field approximation is exact for self-gravitating systems at the thermodynamic limit.

In fact, if we look at the problem more carefully, the argument leading to Eq. (77) is not so obvious because the entropy $S[f]$ has no global maximum, except if we introduce smallscale and large-scale cut-offs. The introduction of these cut-offs is necessary to define correctly the density of state (73), otherwise the integral would diverge (Padmanabhan 1990). The divergence at large scales is associated to the fact that a stellar system has the tendency to evaporate under the effect of encounters. In reality, stellar systems are not allowed to extend to infinity because they interact with the surrounding. For example, globular clusters are subject to the tides of a nearby galaxy so that the Boltzmann distribution has to be truncated at high energies. The Michie-King model,

$f=\left\{\begin{array}{cc}A\left(\mathrm{e}^{-\beta \epsilon}-\mathrm{e}^{-\beta \epsilon_{m}}\right) & \epsilon<\epsilon_{m}, \\ 0 & \epsilon \geq \epsilon_{m},\end{array}\right.$

which is a truncated isothermal, can take into account the evaporation of high energy stars and provides a good description of about $80 \%$ of globular clusters (Binney \& Tremaine 1987). It can be derived from the Fokker-Planck equation (taking into account the encounters between stars) by imposing that the distribution function vanishes at the escape energy $\epsilon=\epsilon_{m}$. In that case, the system is not truly static since it gradually loses stars but we can consider that a globular cluster passes by a succession of quasi-equilibrium configurations. Instead of working with truncated models, it is more convenient to confine the system within a box of radius $R$, the box radius playing the role of a tidal radius. The divergence at small scales of the density of states reflects the natural tendency of a stellar system to form binaries. In this extreme case, the size $a$ of the stars provides a small-scale cut-off. The influence of a small-scale cut-off on the form of the equilibrium phase diagram is discussed by Chavanis (2002c) and Chavanis \& Ispolatov (2002). When the small-scale cut-off $a \rightarrow 0$, which is the limit relevant for globular clusters since the size of the stars is in general much smaller than the interstellar distance, the entropy $S[f]$ possesses two maxima (for sufficiently high energies). The local entropy maximum describes a "gaseous" phase insensitive to the small scale cut-off. It corresponds to the isothermal spheres studied previously. The global entropy maximum is dominated by the small scale cut-off and corresponds to a "condensed" phase made of a few stars close together (or simply a single binary) surrounded by a diffuse halo. This "core-halo" structure is the most probable configuration of a stellar system so it is expected to be reached at equilibrium. Since the observed globular clusters, described by the Michie-King model, do not possess this corehalo structure, we conclude that they are not in true equilibrium but that they are slowly evolving in that direction. However, the formation of binaries can take extremely long times, much larger than the age of the universe. Indeed, equilibrium statistical mechanics tells nothing about the relaxation time. For the timescales contemplated in astrophysics, globular clusters can 
be considered as metastable equilibrium states corresponding to local entropy maxima. Indeed, the probability of transition from a gaseous state to a condensed state (corresponding to the formation of a binary) is extremely small (Katz \& Okamoto 2000; Chavanis \& Ispolatov 2002). Therefore, in the calculation of the density of states (73), we must discard by hands the states which correspond to collapsed configurations (binaries) since they cannot be reached in the timescales of interest. Accordingly, Eq. (77) is correct with $f_{*}$ being the local maximum of $S[f]$. This is precisely the situation studied in Sects. 2 and 3. In fact, this picture is correct only for globular clusters with high energy $E$, well above the Antonov limit $E_{\mathrm{c}}$. Because of evaporation, the energy of a globular cluster slowly decreases until it reaches the point at which the system cannot be in equilibrium anymore (e.g., Katz 1980). At that point, an isothermal sphere becomes unstable and the system undergoes a gravitational collapse (gravothermal catastrophe). The evolution proceeds self-similarly with the formation of a high density core with a shrinking radius and a small mass (Hénon 1961; Cohn 1980; Lynden-Bell \& Eggleton 1980). This core collapse concerns typically $20 \%$ of globular clusters. In theory, the central density becomes infinite in a finite time. In practice, the formation of binaries can release sufficient energy to stop the collapse (Hénon 1961) and even drive a reexpansion of the system (Inagaki \& Lynden-Bell 1983). Then, a series of gravothermal oscillations should follow (Bettwieser \& Sugimoto 1984).

In the canonical ensemble, the object of interest is the partition function

$Z(\beta)=\int \mathrm{e}^{-\beta H} \prod_{i=1}^{N} \mathrm{~d}^{3} \boldsymbol{r}_{i} \mathrm{~d}^{3} \boldsymbol{v}_{i}$.

The free energy is defined by

$F(\beta)=-\frac{1}{\beta} \ln Z$.

The partition function is related to the density of states by the Laplace transform

$Z(\beta)=\int_{-\infty}^{+\infty} \mathrm{d} E g(E) \mathrm{e}^{-\beta E}$.

Using Eq. (76), it can be rewritten

$Z(\beta)=\sum_{i} \mathrm{e}^{S_{i}-\beta E_{i}}=\sum_{i} \mathrm{e}^{J_{i}}$,

where $J_{i}=S_{i}-\beta E_{i}$ is the Massieu function and the sum runs over all macrostates with mass $M$. In the thermodynamic limit, the partition function is dominated by the contribution of the macrostate which maximizes the Massieu function $J_{i} \equiv J[f]$ at fixed mass. In this limit, the mean field approximation is exact and we have

$Z(\beta) \simeq \mathrm{e}^{J\left[f_{*}\right]}, \quad F=E-T S$.

Again, the Massieu function $J[f]$ has no global maximum in the absence of cut-off. If we introduce a small scale cut-off $a$ and a large scale cut-off $R$ and consider the limit $a / R \ll 1$, it is found that the Massieu function $J[f]$ has two maxima (for sufficiently high temperatures). The local maximum corresponds to isothermal "gaseous" configurations and the global maximum to a "condensed" structure in which all the particles are packed together (Aronson \& Hansen 1972; Chavanis 2002c; Chavanis \& Ispolatov 2002). As $a \rightarrow 0$, the density profile of the condensed state tends to a Dirac peak (Kiessling 1989). As in the microcanonical ensemble, the "gaseous" configurations are metastable but they can be long lived and relevant for astrophysical purposes. This justifies why we only select the contribution of the local maximum of $J[f]$ in Eq. (83). This treatment is, however, valid only for sufficiently high temperatures. Below $T_{\mathrm{c}}$, a phase transition similar to the gravothermal catastrophe occurs in the canonical ensemble and leads to a condensed structure containing almost all the mass. The inequivalence of statistical ensembles regarding the formation of binaries (in MCE) or Dirac peak (in CE) is further discussed in Appendices A and B of Sire \& Chavanis (2002).

Finally, the grand canonical partition function is defined by

$Z_{\mathrm{GC}}=\sum_{N=0}^{+\infty} \frac{z^{N}}{N !} \int \prod_{i=1}^{N} \mathrm{~d}^{3} \boldsymbol{r}_{i} \mathrm{~d}^{3} \boldsymbol{v}_{i} \mathrm{e}^{-\beta H_{N}}$,

where $z$ is the fugacity. Applying the standard HubbardStratanovich transformation, it can be rewritten in the form of a path integral (Horwitz \& Katz 1978; Padmanabhan 1991; de Vega \& Sanchez 2002)

$$
\begin{aligned}
& Z_{\mathrm{GC}}=\int \mathcal{D} \phi \mathrm{e}^{-\frac{1}{k T_{\mathrm{eff}}} \int \mathrm{d}^{3} \boldsymbol{r}\left\{\frac{1}{2}(\nabla \phi)^{2}-\mu^{2} \mathrm{e}^{\phi}\right\}}, \\
& T_{\mathrm{eff}}=4 \pi \frac{G m^{2}}{T}, \quad \mu^{2}=\sqrt{\frac{2}{\pi}} z G m^{7 / 2} \sqrt{T},
\end{aligned}
$$

for the Liouville action

$$
A[\phi]=-\frac{1}{k T_{\mathrm{eff}}} \int \mathrm{d}^{3} \boldsymbol{r}\left\{\frac{1}{2}(\nabla \phi)^{2}-\mu^{2} \mathrm{e}^{\phi}\right\} .
$$

It should be emphasized that $\phi$ is a formal field which is not the gravitational field. In the mean-field approximation, the path integral is dominated by the contribution of the field $\phi_{0}$ which maximizes the Liouville action (87). The cancellation of the first order variations gives

$\Delta \phi_{0}=-\mu^{2} \mathrm{e}^{\phi_{0}}$,

which is similar to the Boltzmann-Poisson Eq. (12). This allows one to identify $\phi_{0}$ with the equilibrium gravitational potential $\Phi$ (up to a negative proportionality factor). The second order variations of $A$ at the critical point must be negative for $A\left[\phi_{0}\right]$ to be a maximum. This yields the condition

$\int \mathrm{d}^{3} \boldsymbol{r} \delta \phi\left\{\Delta+\mu^{2} \mathrm{e}^{\phi_{0}}\right\} \delta \phi \leq 0$,

which is equivalent to the maximization of the grand potential $G$ (see Sect. 3.1). Therefore, the thermodynamical approach is equivalent to the statistical mechanics or field theory approach. We note, however, that the Liouville action $A[\phi]$ is not the same functional as the grand potential $G[\rho]$ although they give the same critical points and the same conditions of stability. 


\section{Generalized thermodynamics and Tsallis entropy}

\subsection{General considerations}

It has been recently argued that the classical Boltzmann entropy may not be relevant for non-extensive systems and that Tsallis entropies, also called $q$-entropies, should be used instead. These entropies can be written

$S_{q}=-\frac{1}{q-1} \int\left(f^{q}-f\right) \mathrm{d}^{3} r \mathrm{~d}^{3} \boldsymbol{v}$

where $q$ is a real number. For $q \rightarrow 1$, this expression returns the classical Boltzmann entropy (4) as a particular case.

The first application of Tsallis generalized thermodynamics in stellar systems was made by Plastino \& Plastino (1997) who showed that the extremization of Tsallis entropy leads to stellar polytropes with an index

$n=\frac{3}{2}+\frac{1}{q-1}$

For $q \rightarrow 1$, or $n \rightarrow+\infty$, we recover isothermal spheres. For $q>9 / 7$ (i.e. $n<5$ ), the density drops to zero at a finite distance so that the mass is finite. Then, Taruya \& Sakagami (2002a) considered the stability problem in the microcanonical ensemble by extending Padmanabhan's classical analysis of the Antonov instability to the case of polytropic distribution functions. A configuration is stable in the sense of generalized thermodynamics if it corresponds to a maximum of Tsallis entropy at fixed mass and energy. They considered polytropic spheres confined within a box and showed that polytropes with index $n \geq 5$ become unstable above a certain density contrast while polytropes with $n \leq 5$ are always stable. On the other hand, if we consider the canonical ensemble, we have to select maxima of Tsallis free energy $J_{q}=S_{q}-\beta E$ at fixed mass and $\beta$ (the generalized inverse temperature). It is found (Taruya \& Sakagami 2002b; see also Sect. 5.5) that polytropes with index $n \geq 3$ become unstable above a certain density contrast while polytropes with $n \leq 3$ are always stable. In our sense, the main interest of the $q$-entropies (and their attractive nature) is to offer a simple generalization of classical thermodynamics which leads to models that are still analytically tractable since exponentials are replaced by power laws. This approach is interesting to develop, at least formally, as it offers a nice connexion between polytropic and isothermal spheres, which are the most popular mathematical models of self-gravitating systems. We shall therefore devote a section to this generalization. However, in Sect. 7, we shall argue that Tsallis entropies are just a particular case of $H$-functions $S[f]$ whose maximization at fixed mass and energy determines nonlinearly stable stationary solutions of the Vlasov equation (Tremaine et al. 1986). We shall also argue that the maximization of the $J$-function $J[f]=S-\beta E$ at fixed mass determines nonlinearly stable stationary solutions of the Euler-Jeans equations. This discussion should demystify the concept of generalized thermodynamics introduced by Tsallis (1988).

\subsection{Stellar polytropes}

The extremization of Tsallis entropy at fixed mass and energy yields (Plastino \& Plastino 1997; Taruya \& Sakagami 2002a)

$f=A\left[\alpha-\Phi-\frac{v^{2}}{2}\right]^{\frac{1}{q-1}}$,

$A=\left[\frac{(q-1) \beta}{q}\right]^{\frac{1}{q-1}}, \quad \alpha=\frac{1+(q-1)(\mu / T)}{(q-1) \beta}$,

where $\beta=1 / T$ and $\mu / T$ (related to $\alpha$ ) are Lagrange multipliers which can be called inverse temperature and chemical potential in the generalized sense. The distribution function (92) corresponds to stellar polytropes that were first introduced by Plummer (1911). Their derivation from a variational principle was discussed by Ipser (1974) among others. Note that Eq. (92) is valid for $v^{2} \leq 2(\alpha-\Phi)$. For $v^{2} \geq 2(\alpha-\Phi)$, we set $f=0$. The spatial density $\rho=\int f \mathrm{~d}^{3} \boldsymbol{v}$ and the pressure $p=\frac{1}{3} \int f v^{2} \mathrm{~d}^{3} \boldsymbol{v}$ can be expressed as

$\rho=4 \sqrt{2} \pi A(\alpha-\Phi)^{n} B(3 / 2, n-1 / 2)$,

$p=4 \sqrt{2} \pi A(\alpha-\Phi)^{n+1} \frac{1}{n+1} B(3 / 2, n-1 / 2)$,

with $B(a, b)$ being the $\beta$ function and use has been made of Eq. (91). Furthermore, in obtaining Eq. (95), we have used the identity $B(m+1, n)=m B(m, n) /(m+n)$. The integrability condition $\int f \mathrm{~d}^{3} \boldsymbol{v}<\infty$ requires that $n>1 / 2$. Eliminating the gravitational potential between the relations (94) and (95), we recover the well-known fact that stellar polytropes satisfy the equation of state

$p=K \rho^{\gamma}, \quad \gamma=1+\frac{1}{n}$,

like gaseous polytropes (note, however, that these systems do not have the same phase space distribution function, unlike isothermal spheres). In the present context, the polytropic constant is given by

$K=\frac{1}{(n+1)}\{4 \sqrt{2} \pi A B(3 / 2, n-1 / 2)\}^{-1 / n}$.

Using Eqs. (92) and (94), the distribution function can be written as a function of the density as

$f=\frac{1}{Z}\left[\rho^{1 / n}-\frac{v^{2} / 2}{(n+1) K}\right]^{n-3 / 2}$,

with

$Z=4 \sqrt{2} \pi B(3 / 2, n-1 / 2)[K(n+1)]^{3 / 2}$.

Using the foregoing relations, it is possible to express the total energy and the entropy in terms of $\rho$ and $p$ according to (Taruya \& Sakagami 2002b; see also Sect. 7.8)

$E=\frac{3}{2} \int p \mathrm{~d}^{3} \boldsymbol{r}+\frac{1}{2} \int \rho \Phi \mathrm{d}^{3} \boldsymbol{r}$

$S=-\left(n-\frac{3}{2}\right)\left\{\frac{1}{T} \int p \mathrm{~d}^{3} \boldsymbol{r}-M\right\}$. 


\subsection{The Lane-Emden equation}

The equilibrium structure of a polytrope is determined by substituting the relation (94) between $\rho$ and the gravitational potential $\Phi$ inside the Poisson Eq. (3). This is equivalent to using the condition of hydrostatic equilibrium $\nabla p=-\rho \nabla \Phi$ with the equation of state (96). Letting

$\rho=\rho_{0} \theta^{n}, \quad \xi=\left[\frac{4 \pi G \rho_{0}^{1-1 / n}}{K(n+1)}\right]^{1 / 2} r$,

where $\rho_{0}$ is the central density, we can reduce the condition of hydrostatic equilibrium to the Lane-Emden equation (Chandrasekhar 1942)

$\frac{1}{\xi^{2}} \frac{\mathrm{d}}{\mathrm{d} \xi}\left(\xi^{2} \frac{\mathrm{d} \theta}{\mathrm{d} \xi}\right)=-\theta^{n}$

with boundary conditions $\theta(0)=1, \theta^{\prime}(0)=0$.

For $n<5$, the density vanishes at some radius identified with the radius of the polytrope (in terms of the normalized distance, this corresponds to $\theta=0$ at $\xi=\xi_{1}$ ). Such polytropic configurations (with infinite density contrasts) will be refered to as complete polytropes. We shall also consider the case of incomplete polytropes, with arbitrary index, enclosed within a box of radius $R$. For these configurations, the Lane-Emden equation must be solved between $\xi=0$ and $\xi=\alpha$ with

$\alpha=\left[\frac{4 \pi G \rho_{0}^{1-1 / n}}{K(n+1)}\right]^{1 / 2} R$.

We also recall the expression of the Milne variables that will be used in the sequel,

$u=-\frac{\xi \theta^{n}}{\theta^{\prime}}, \quad v=-\frac{\xi \theta^{\prime}}{\theta}$.

These variables satisfy the identities

$\frac{1}{u} \frac{\mathrm{d} u}{\mathrm{~d} \xi}=\frac{1}{\xi}(3-n v-u)$,

$\frac{1}{v} \frac{\mathrm{d} v}{\mathrm{~d} \xi}=\frac{1}{\xi}(u+v-1)$

which can be derived from Eq. (103). The phase portrait of polytropes in the $(u, v)$ plane and the properties that we shall need in the following are summarized in Chavanis (2002b).

\subsection{Turning point criterion}

Eliminating $A$ between Eqs. (93) and (97), we find that

$\beta=\left[\frac{4 \pi Q^{n-1}}{K^{n}}\right]^{\frac{2}{2 n-3}}$,

where we have defined (Taruya \& Sakagami 2002b)

$Q=\left\{\frac{\left(n-\frac{1}{2}\right)^{n-\frac{3}{2}}}{16 \sqrt{2} \pi^{2} B(3 / 2, n-1 / 2)(n+1)^{n}}\right\}^{\frac{1}{n-1}}$.

In a preceding paper (Chavanis 2002b), we have established that the mass of the configuration is related to the central density $\rho_{0}$ (through the parameter $\alpha$ ) by the relation

$\eta \equiv \frac{M}{4 \pi}\left[\frac{4 \pi G}{K(1+n)}\right]^{\frac{n}{n-1}} \frac{1}{R^{\frac{n-3}{n-1}}}=\left(u_{0} v_{0}^{n}\right)^{\frac{1}{n-1}}$.
We had conjectured that $\eta$ plays the role of a temperature in the context of Tsallis generalized thermodynamics. This is indeed the case since, using Eq. (108), $\eta$ can be expressed in terms of $\beta$ as

$\eta=\frac{1}{Q}\left\{\frac{G^{n} M^{n-1}}{R^{n-3}} \beta^{n-\frac{3}{2}} \frac{1}{(n+1)^{n}}\right\}^{\frac{1}{n-1}}$.

For $n \rightarrow+\infty$, this parameter becomes equivalent to the parameter $\eta=\beta G M / R$ introduced in the study of isothermal spheres (Chavanis 2002a).

In the framework of generalized thermodynamics, the parameter conjugate to the energy with respect to Tsallis entropy $S_{q}$ is the inverse temperature $\beta$ (microcanonical description) and the parameter conjugate to the inverse temperature with respect to Tsallis free energy $J_{q}=S_{q}-\beta E$ is $-E$ (canonical description). The $E-T$ curve has been plotted and discussed in Chavanis (2002b) for different values of the polytropic index. According to the turning point criterion, the series of equilibria becomes unstable in the canonical ensemble for $\mathrm{d} \eta / \mathrm{d} \alpha=0$. We have shown that this condition is equivalent to

$u_{0}=\frac{n-3}{n-1}=u_{\mathrm{s}}$.

For $n \leq 3$, the polytropes are always stable since the $\eta(\alpha)$ curve is monotonic. The polytropes start to be unstable (for sufficiently high density contrasts) when $n>3$. This generalized thermodynamical stability criterion coincides with the Jeans dynamical stability criterion based on the Navier-Stokes equations for a polytropic gas (Chavanis 2002b). Since $K$ is considered as a constant when we analyze the stability of polytropic spheres with respect to the Navier-Stokes equations, and since $K$ is related to the temperature via Eq. (108), this corresponds to a sort of "canonical situation". This explains why the condition of dynamical stability for gaseous polytropes is equivalent to the condition of thermodynamical stability for stellar polytropes in the canonical ensemble. This equivalence is further discussed in Sect. 7.

We now turn to the grand canonical situation. Combining Eqs. (94), (93) and (109), we can write the density field as

$\rho=\frac{Q^{1-n}}{4 \pi(n+1)^{n} \beta^{3 / 2}}\left[n-\frac{3}{2}+\frac{\mu}{T}-\beta \Phi\right]^{n}$.

This expression suggests to define a generalized fugacity by the relation $n z^{1 / n} \equiv n-3 / 2+\mu / T$ so that $z$ is equivalent to the usual fugacity $\mathrm{e}^{\frac{\mu}{T}}$ when $n \rightarrow+\infty$. Taking $r=R$ in Eq. (113) and comparing with Eq. (102), we get

$\rho_{0} \theta(\alpha)^{n}=\frac{Q^{1-n}}{4 \pi(n+1)^{n} \beta^{3 / 2}}\left(n z^{1 / n}+\frac{\beta G M}{R}\right)^{n}$,

where we have used $\Phi(R)=-G M / R$. Expressing the central density in terms of $\alpha$, using Eqs. (104) and (108), we find

$\rho_{0}=\frac{Q}{4 \pi}\left[\frac{(n+1)^{n} \alpha^{2 n}}{G^{n} R^{2 n} \beta^{n-\frac{3}{2}}}\right]^{\frac{1}{n-1}}$.

From Eqs. (114) and (115), we get

$n z^{1 / n}=Q\left[\frac{(n+1)^{n} \beta^{1 / 2}}{G R^{2}}\right]^{\frac{1}{n-1}} \alpha^{\frac{2}{n-1}} \theta(\alpha)-\frac{\beta G M}{R}$. 


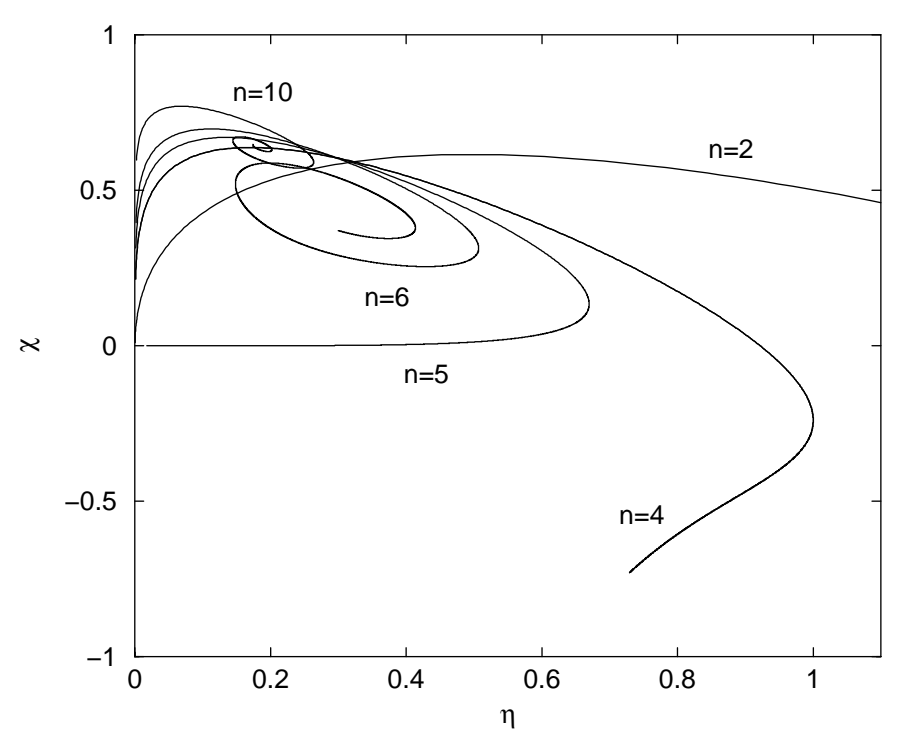

Fig. 15. Fugacity vs. mass (for a given temperature) in the framework of generalized thermodynamics for different polytropic index. For $1<$ $n \leq 3, \chi$ has one turning point but not $\eta$; for $3<n \leq 5$, both $\chi$ and $\eta$ have one turning point; for $n>5, \chi$ and $\eta$ have an infinity of turning points. For $n<5$, complete polytropes correspond to the terminal point of the $\eta-\chi$ curve. For $n>5$, the curve has a spiral behaviour towards the limit point $\left(\eta_{\mathrm{s}}, \chi_{\mathrm{s}}\right)$ corresponding to the singular sphere $\left(u_{\mathrm{s}}, v_{\mathrm{s}}\right)=\left(\frac{n-3}{n-1}, \frac{2}{n-1}\right)$.

Eliminating $M$ thanks to Eq. (111) and introducing the Milne variables (105), we finally obtain

$\chi \equiv \frac{n}{Q} z^{1 / n}\left[\frac{G R^{2}}{(n+1)^{n} \beta^{1 / 2}}\right]^{\frac{1}{n-1}}=\left(u_{0} v_{0}\right)^{\frac{1}{n-1}}\left(1-v_{0}\right)$.

For $n \rightarrow+\infty$, this parameter becomes equivalent to the one introduced in Sect. 2.4 for isothermal spheres (note that for sufficiently small index $n$, the parameter $\chi$ can become negative when $v_{0}>1$; therefore, the definition of $z$ given previously is not valid for small $n$ ). According to the turning point criterion, the series of equilibria becomes unstable in the grand canonical ensemble when $\mathrm{d} \chi / \mathrm{d} \alpha=0$. This gives the condition

$2\left(1-v_{0}\right)-(n-1) u_{0} v_{0}=0$.

The values of $\alpha$ at which $\chi$ is extremum can be determined by a graphical construction like in Chavanis (2002b). They correspond to the intersections between the hyperbole defined by Eq. (118) and the solution curve in the $(u, v)$ plane (which depends on the index $n$ ). From these graphical constructions, we deduce that the function $\chi(\alpha)$ has one maximum for $n \leq 5$. For $n=5$, the Lane-Emden equation can be solved analytically and we get

$\chi=\frac{\alpha^{4}}{\left(1+\frac{1}{3} \alpha^{2}\right)^{12}}$,

which is maximum for $\alpha=(3 / 5)^{1 / 2}$. For $n>5$, the function $\chi(\alpha)$ has an infinite number of extrema. The onset of gravitational instability corresponds to the first maximum. The fugacity vs. mass plot (for a given temperature) can be deduced from
Fig. 15. Collecting the results obtained by different authors, incomplete polytropes with high density contrasts are unstable in MCE for $n>5$, in CE for $n>3$ and in GCE for all $n$. Complete polytropes are always stable in MCE, stable in $\mathrm{CE}$ only for $n<3$ and never stable in GCE.

In the following, we analyze the stability of polytropic spheres by explicitly calculating the second order variations of the thermodynamical potential. This study has been done by Taruya \& Sakagami (2002a, b) in MCE and CE. We shall consider CE in relation with our previous study (Chavanis 2002b), GCE and GMCE.

\subsection{Generalized thermodynamical stability in the canonical ensemble}

In the canonical ensemble, the thermodynamical parameter is the free energy (Massieu function) $J=S-\frac{1}{T} E$ which is explicitly given by

$J=-\frac{n}{T} \int p \mathrm{~d}^{3} \boldsymbol{r}-\frac{1}{2 T} \int \rho \Phi \mathrm{d}^{3} \boldsymbol{r}$,

where we have used Eqs. (100) and (101). Using the equation of state (96), the second order variations of free energy can be put in the form

$\delta^{2} J=-\frac{1}{2 T}\left\{\int \frac{n+1}{n} \frac{p}{\rho^{2}}(\delta \rho)^{2} \mathrm{~d}^{3} \boldsymbol{r}+\int \delta \rho \delta \Phi \mathrm{d}^{3} \boldsymbol{r}\right\}$.

As in previous papers, we introduce the function $q(r)$ defined by

$\delta \rho=\frac{1}{4 \pi r^{2}} \frac{\mathrm{d} q}{\mathrm{~d} r}$,

and satisfying the perturbed Gauss theorem

$\frac{\mathrm{d} \delta \Phi}{\mathrm{d} r}=\frac{G q}{r^{2}}$

The conservation of mass imposes $q(0)=q(R)=0$. After an integration by parts, we can rewrite Eq. (121) in the form

$\delta^{2} J=\frac{1}{2 T} \int_{0}^{R} \mathrm{~d} r q\left[K \gamma \frac{\mathrm{d}}{\mathrm{d} r}\left(\frac{\rho^{\gamma-2}}{4 \pi r^{2}} \frac{\mathrm{d}}{\mathrm{d} r}\right)+\frac{G}{r^{2}}\right] q$.

The point of marginal stability is therefore determined by the condition

$K \gamma \frac{\mathrm{d}}{\mathrm{d} r}\left(\frac{\rho^{\gamma-2}}{4 \pi r^{2}} \frac{\mathrm{d} q}{\mathrm{~d} r}\right)+\frac{G q}{r^{2}}=0$.

This is the same equation as that determining the dynamical stability of gaseous polytropes (Chavanis 2002b). We have solved this equation and showed that the boundary condition $q(R)=0$ returns the condition of Eq. (112) brought by the turning point criterion. The structure of the density perturbation profile that triggers instability is also discussed in our previous paper. 


\subsection{Generalized thermodynamical stability in the grand canonical ensemble}

The thermodynamical potential in the grand canonical ensemble is $G=S-\frac{1}{T} E+\frac{\mu}{T} M$, i.e.

$G=n z^{1 / n} \int \rho \mathrm{d}^{3} \boldsymbol{r}-\frac{n}{T} \int p \mathrm{~d}^{3} \boldsymbol{r}-\frac{1}{2 T} \int \rho \Phi \mathrm{d}^{3} \boldsymbol{r}$.

Its second order variations are given by Eq. (121) like for the free energy. Only the boundary conditions are different since the mass is not conserved anymore. Introducing the function $X(r)$ defined by Eq. (31), we obtain after an integration by parts

$\delta^{2} G=\frac{1}{2 T G^{2}} \int_{0}^{R} \mathrm{~d} r X^{\prime}\left[G+K \gamma \frac{\mathrm{d}}{\mathrm{d} r}\left(\frac{\rho^{\gamma-2}}{4 \pi} \frac{\mathrm{d}}{\mathrm{d} r}\right)\right] X^{\prime}$.

The point of marginal stability is determined by the equation

$K \gamma \frac{\mathrm{d}}{\mathrm{d} r}\left(\frac{\rho^{\gamma-2}}{4 \pi} X^{\prime \prime}\right)+G X^{\prime}=0$

with the boundary conditions (32). Integrating once, we get

$K \gamma \frac{\rho^{\gamma-2}}{4 \pi} X^{\prime \prime}+G X=0$.

In terms of the dimensionless variables introduced in Sect. 5.3, we have to solve the problem

$\frac{1}{n} \theta^{1-n} X^{\prime \prime}+X=0$,

$X(0)=X^{\prime}(\alpha)=0$.

Let us introduce the differential operator

$\mathcal{L}=\frac{1}{n} \theta^{1-n} \frac{\mathrm{d}^{2}}{\mathrm{~d} \xi^{2}}+1$.

Using the Lane-Emden Eq. (103), it is easy to check that

$\mathcal{L}(\xi)=\xi, \quad \mathcal{L}\left(\xi^{2} \theta^{\prime}\right)=-\frac{2}{n} \xi \theta, \quad \mathcal{L}(\xi \theta)=\frac{n-1}{n} \xi \theta$.

Therefore, the general solution of Eq. (130) satisfying $X(0)=0$ is

$X=\xi\left(\xi \theta^{\prime}+\frac{2}{n-1} \theta\right)$.

The critical value of $\alpha$ at which the series of equilibria becomes unstable in GCE is determined by the boundary condition $X^{\prime}(\alpha)=0$. Using Eq. (134) and introducing the Milne variables (105), we find that this condition is equivalent to Eq. (118) in agreement with the turning point criterion. The density perturbation profile triggering instability can be deduced from Eqs. (47) and (134). We get

$\frac{\delta \rho}{\rho}=\frac{3}{4 \pi} c_{1}\left(v_{\mathrm{s}}-v\right)$,

with $v_{\mathrm{s}}=2 /(n-1)$. Equation (135) coincides with the expression found in CE. The number of nodes of the perturbation profile can be determined by a graphical construction like in Chavanis (2002b). First, consider the case $n=5$ which can be solved analytically. The solution of the equation $v(\xi)=v_{\mathrm{s}}$ is $\xi_{(1)}=\sqrt{3}$. Since $\xi_{(1)}>\alpha=(3 / 5)^{1 / 2}$, we conclude that the perturbation profile in GCE has no node contrary to the equivalent situation in $\mathrm{CE}$. This conclusion remains true for all index $n \leq 5$. Indeed, the critical case $\xi_{(1)}=\alpha$ would occur for an index $n$ such that $\left(u_{\mathrm{s}}, v_{\mathrm{s}}\right)$ belongs to the solution curve (substitute $v_{0}=v_{\mathrm{s}}$ in Eq. (118)). This is never the case for $n \leq 5$ (see Chandrasekhar 1942). Therefore, the structure of the density profile for $n<5$ is the same as for $n=5$ so it has no node. For $n>5$, the first mode of instability has no node, the second mode of instability one node etc.

\subsection{Generalized thermodynamical stability in the grand microcanonical ensemble}

In the grand microcanonical ensemble, the fixed parameters are the fugacity and the energy. The normalized energy is given by (Taruya \& Sakagami 2002a)

$\Lambda \equiv-\frac{E R}{G M^{2}}=-\frac{1}{n-5}\left[\frac{3}{2}\left(1-\frac{1}{v_{0}}\right)+\frac{n-2}{n+1} \frac{u_{0}}{v_{0}}\right]$

Eliminating $\beta$ in Eq. (117) in profit of $E$, using Eqs. (111) and (136), we find that the relevant control parameter

$v \equiv-\frac{\left(n z^{1 / n}\right)^{2(2 n-3)}}{(n+1)^{4 n}} \frac{1}{Q^{4(n-1)}} G^{5} R^{7} E$,

is related to $\alpha$ by

$\begin{aligned} v= & -u_{0}^{4} v_{0}^{6}\left(1-v_{0}\right)^{2(2 n-3)} \\ & \times \frac{1}{n-5}\left[\frac{3}{2}\left(1-\frac{1}{v_{0}}\right)+\frac{n-2}{n+1} \frac{u_{0}}{v_{0}}\right] .\end{aligned}$

In the grand microcanonical ensemble, we have to maximize the thermodynamical potential $\mathcal{K}=S+\frac{\mu}{T} M$ at fixed $E$ and $\mu / T$. The entropy and the energy are expressed in terms of $\rho$ and $K$ by Eqs. (100), (101) and (96). We vary the density and use the energy constraint to determine the corresponding variation of $K$ (which is related to the inverse temperature $\beta$ ). After some algebra, we find that the second order variations of $\mathcal{K}$ are given by

$$
\begin{aligned}
\delta^{2} \mathcal{K}= & -\frac{1}{2} \int \delta \rho \delta \Phi \mathrm{d}^{3} \boldsymbol{r}-\frac{1}{2} \gamma \int \frac{p}{\rho^{2}}(\delta \rho)^{2} \mathrm{~d}^{3} \boldsymbol{r} \\
& -\frac{2 n}{3(2 n-3)} \frac{1}{\int p \mathrm{~d}^{3} \boldsymbol{r}}\left[\int\left(\Phi+\frac{3 \gamma p}{2 \rho}\right) \delta \rho \mathrm{d}^{3} \boldsymbol{r}\right]^{2} .
\end{aligned}
$$

(To simplify the formulae, we have not written the positive proportionality factor in front of $\delta^{2} \mathcal{K}$.) Introducing the variable $X$ defined previously and integrating by parts, we can put the problem in the form

$\delta^{2} \mathcal{K}=\int_{0}^{R} \mathrm{~d} r \int_{0}^{R} \mathrm{~d} r^{\prime} X^{\prime}(r) K\left(r, r^{\prime}\right) X^{\prime}\left(r^{\prime}\right)$,

with

$\begin{aligned} K\left(r, r^{\prime}\right)= & \left\{G+\gamma \frac{\mathrm{d}}{\mathrm{d} r}\left(\frac{p}{4 \pi \rho^{2}} \frac{\mathrm{d}}{\mathrm{d} r}\right)\right\} \delta\left(r-r^{\prime}\right)-\frac{4 n}{3(2 n-3)} \\ & \times \frac{1}{\int p \mathrm{~d}^{3} \boldsymbol{r}}\left[\left(\Phi+\frac{3 \gamma p}{2 \rho}\right) r\right]^{\prime}(r)\left[\left(\Phi+\frac{3 \gamma p}{2 \rho}\right) r\right]^{\prime}\left(r^{\prime}\right) .\end{aligned}$ 
The condition of marginal stability reads

$$
\begin{aligned}
G X+\frac{\gamma p}{4 \pi \rho^{2}} X^{\prime \prime}= & \frac{4 n}{3(2 n-3)} \frac{1}{\int p \mathrm{~d}^{3} \boldsymbol{r}}\left(\Phi+\frac{3 \gamma p}{2 \rho}\right) r \\
& \times \int_{0}^{R}\left[\left(\Phi+\frac{3 \gamma p}{2 \rho}\right) r\right]^{\prime} X^{\prime} \mathrm{d} r
\end{aligned}
$$

We now need to relate the gravitational potential $\Phi$ to the Lane-Emden function $\theta$. Integrating the equation of hydrostatic equilibrium for a polytropic equation of state, we readily find that

$(n+1) \frac{p}{\rho}=-\Phi+$ const.

The constant can be determined from the boundary condition $\Phi(R)=-G M / R$. Using the relation

$\frac{G M}{R}=-(n+1) K \rho_{0}^{1 / n} \alpha \theta^{\prime}(\alpha)$

which results from Eqs. (110) and (104), we find that Eq. (143) can be written

$\Phi=(n+1) K \rho_{0}^{1 / n}\left(\theta(\alpha)-\theta+\alpha \theta^{\prime}(\alpha)\right)$.

Inserting this relation in Eq. (142) and introducing the dimensionless variables defined in Sect. 5.3, we find that

$$
\begin{aligned}
X & +\frac{1}{n} \theta^{1-n} X^{\prime \prime}=\frac{2(n+1) \xi}{\int_{0}^{\alpha} \theta^{1+n} \xi^{2} \mathrm{~d} \xi}\left[\theta(\alpha)+\frac{3-2 n}{2 n} \theta+\alpha \theta^{\prime}(\alpha)\right] \\
& \times\left\{-\frac{1}{3} \int_{0}^{\alpha} \xi \theta^{n} X \mathrm{~d} \xi+\frac{\alpha \theta^{\prime}(\alpha)+\theta(\alpha)}{2 n-3} X(\alpha)\right\},
\end{aligned}
$$

where we have integrated by parts and used the identity $(\xi \theta)^{\prime \prime}=$ $-\xi \theta^{n}$ equivalent to the Lane-Emden Eq. (103). We are led therefore to solve a problem of the form

$\frac{1}{n} \theta^{1-n} X^{\prime \prime}+X=V \theta \xi+W \xi$

$X(0)=X^{\prime}(\alpha)=0$.

Using the identities (133), we find that the general solution of Eq. (147) satisfying $X(0)=0$ is

$X=W \xi+b\left[\xi \theta+\frac{1}{2}(n-1) \xi^{2} \theta^{\prime}\right]-\frac{n}{2} V \xi^{2} \theta^{\prime}$

The constant $b$ is determined by the boundary condition $X^{\prime}(\alpha)=0$. Substituting the resulting expression for $X(\xi)$ in Eq. (146), and using the identities of Appendix B, we obtain after tedious algebra a condition for $\alpha$ which is equivalent to the one obtained from the turning point argument, i.e. by setting $\mathrm{d} v / \mathrm{d} \alpha=0$.

\section{The isobaric ensemble}

We now consider the stability of isothermal spheres in contact with a medium exerting a constant pressure $P$ on their boundary. In this isobaric ensemble, the volume of the system can change unlike in the Antonov problem. This situation was first considered by Ebert (1955), Bonnor (1956) and McCrea (1957) who found that an isothermal gas becomes gravitationally unstable above a critical pressure $P_{\max }$ or above a maximum compression. We shall complete these results by supplying an entirely analytical derivation of the stability criterion and by showing the equivalence between thermodynamical and dynamical stability (Jeans problem). We shall also consider the case of polytropic gas spheres under external pressure.

\subsection{Isothermal gas spheres under external pressure}

Let us consider an isothermal gas sphere with mass $M$ at temperature $T$ and under pressure $P$. The first principle of thermodynamics can be written $\delta S-\frac{1}{T} \delta E-\frac{p}{T} \delta V=0$. In the isothermal-isobaric ensemble (TBE), the relevant thermodynamical potential is the Gibbs energy $\mathcal{G}=S-\frac{1}{T} E-\frac{P V}{T}$. Its first variations satisfy $\delta \mathcal{G}=-E \delta\left(\frac{1}{T}\right)-V \delta\left(\frac{P}{T}\right)$ so that, at statistical equilibrium, the system is in the state that maximizes $\mathcal{G}$ at fixed temperature $T$ and pressure $P$.

For an isothermal gas, $p=\frac{k}{m} \rho T$. Using Eq. (13), the pressure on the boundary of the sphere is

$P=\frac{k T}{m} \rho(R)=\frac{\rho_{0}}{\beta} \mathrm{e}^{-\psi(\alpha)}$.

The central density $\rho_{0}$ can be eliminated via the relation (15). Eliminating the radius $R$ via the relation (20) and introducing the Milne variables (16), we obtain after simplification

$\mathcal{P} \equiv 4 \pi G^{3} \beta^{4} M^{2} P=u_{0} v_{0}^{3}$.

Similarly, the normalized volume of the sphere is given by

$\mathcal{V} \equiv \frac{3 V}{4 \pi \beta^{3} G^{3} M^{3}}=\frac{1}{v_{0}^{3}}$

Using the expansion of the Milne variables for $\alpha \rightarrow 0$,

$u_{0}=3-\frac{1}{5} \alpha^{2}+\ldots, \quad v_{0}=\frac{1}{3} \alpha^{2}-\ldots$,

we find that the first order correction to Boyle's law due to selfgravity is

$P V=N k T-\lambda \frac{G M^{2}}{V^{1 / 3}}$,

with $\lambda=\frac{1}{5}\left(\frac{4}{3} \pi\right)^{1 / 3}$. This formula can also be derived from the Virial theorem

$P V=\int p \mathrm{~d}^{3} \boldsymbol{r}+\frac{1}{3} W$

by using the isothermal equation of state and by assuming that the density $\rho$ is uniform (in which case the potential energy $\left.W=-3 G M^{2} / 5 R\right)$. As reported by Bonnor (1956), Eq. (154) was first suggested by Terletsky (1952) as a simple attempt to take into account gravitational effects in the perfect gas law. It appears here as a systematic expansion in terms of the concentration factor $\alpha$. For large concentrations, Eq. (154) is not valid anymore and Eqs. (151)-(152) must be used instead. The $P-V$ curve is plotted in Fig. 16. It presents a striking spiral behaviour towards the limit point $\left(\mathcal{V}_{\mathrm{s}}, \mathcal{P}_{\mathrm{s}}\right)=(1 / 8,8)$ corresponding to the singular sphere. This is similar to the 


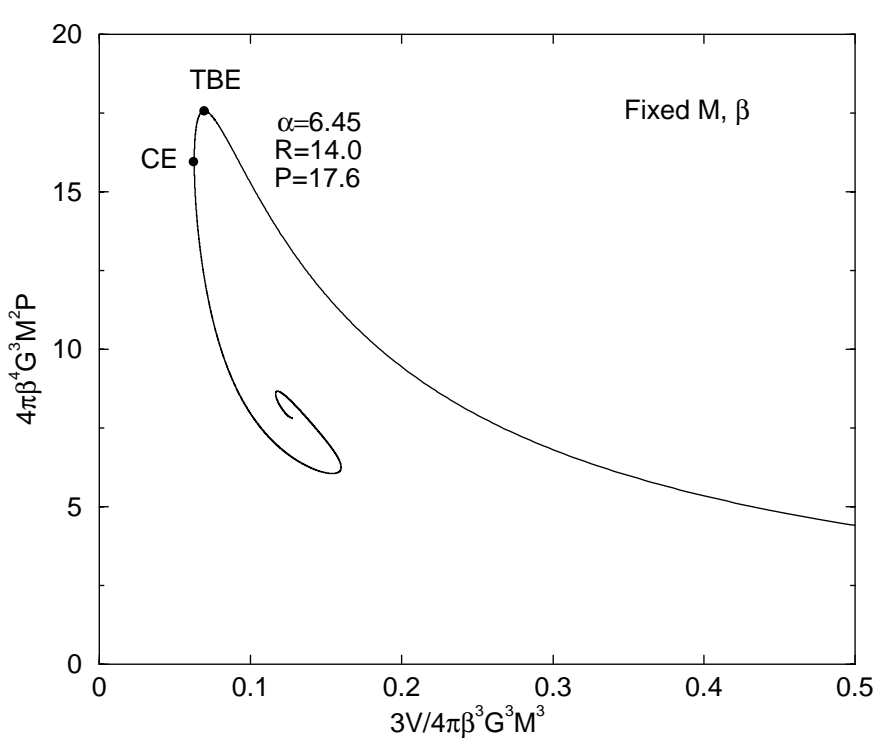

Fig. 16. The $P-V$ diagram for isothermal spheres. The series of equilibria becomes unstable at TBE in the isothermal-isobaric ensemble.

$E-T$ curve and other curves studied in Sect. 2.4. This is interesting to note, for historical reasons, because this diagram was discoved before the fundamental papers of Antonov (1962) and Lynden-Bell \& Wood (1968) on the gravothermal catastrophe. Like the minimum energy of Antonov, there exists a maximum pressure $P_{\max }=1.40 k^{4} T^{4} / G^{3} M^{2} m^{4}$ above which no hydrostatic equilibrium for an isothermal gas is possible. Alternatively, this corresponds to a minimum temperature $T_{\min }=0.919 \mathrm{G}^{3 / 4} \mathrm{mM}^{1 / 2} P^{1 / 4} / \mathrm{k}$ for a given mass and pressure. From the turning point criterion, the series of equilibria becomes unstable in TBE at the point of maximum pressure. The condition $\mathrm{d} \mathcal{P} / \mathrm{d} \alpha=0$ leads to

$2 u_{0}-v_{0}=0$.

The critical value $\alpha$ is determined by the intersection between the spiral in the $(u, v)$ plane and the straight line (156). Its numerical value is $\alpha=6.45$ corresponding to a density contrast of 14.0. More compressed isothermal spheres, ie. those for which $V \leq V_{\min }=0.29 G^{3} M^{3} m^{3} / k^{3} T^{3}$ are thermodynamically unstable.

Using Eqs. (151)-(152) and the identities (17)-(18), we readily obtain

$\frac{\mathrm{d} \ln \mathcal{V}}{\mathrm{d} \alpha}=-\frac{3}{\alpha}\left(u_{0}-1\right)$,

and

$\frac{\mathrm{d} \ln \mathcal{P}}{\mathrm{d} \alpha}=\frac{1}{\alpha}\left(2 u_{0}-v_{0}\right)$.

Therefore, the "compressibility" $\kappa=-(\partial \ln P / \partial \ln V)_{N, T}$ can be expressed as

$\kappa=\frac{1}{3} \frac{2 u_{0}-v_{0}}{u_{0}-1}$

Using $u_{0}=3 P V / N k T$ and $v_{0}=(4 \pi / 3)^{1 / 3} G M m / k T V^{1 / 3}$ according to Eqs. (151) and (152), we can check that Eq. (159) is equivalent to Eq. (2.16) of Bonnor (1956), but it has been obtained here in a much more direct manner. Gravitational instability in TBE occurs when the compressibility becomes negative passing by $\kappa=0$.

We now consider the dynamical stability of isothermal gas spheres under external pressure and show the equivalence with the thermodynamical criterion. This problem was studied numerically by Yabushita (1968) but we provide here an entirely analytical solution. In a preceding paper, we have found that the equation of radial pulsations for a gas with an isothermal equation of state could be written (Chavanis 2002a)

$$
\frac{k}{m} \frac{\mathrm{d}}{\mathrm{d} r}\left(\frac{1}{4 \pi \rho r^{2}} \frac{\mathrm{d} q}{\mathrm{~d} r}\right)+\frac{G q}{T r^{2}}=\frac{\lambda^{2}}{4 \pi \rho T r^{2}} q,
$$

where $\lambda$ is the growth rate of the perturbation (such that $\delta \rho \sim \mathrm{e}^{\lambda t}$ ) and $q$ is defined by Eq. (122). In the isobaric ensemble, the boundary conditions are given by Eq. (C.4) of Appendix C. Introducing dimensionless variables and considering the case of marginal stability $\lambda=0$, we are led to solve the problem

$\frac{\mathrm{d}}{\mathrm{d} \xi}\left(\frac{\mathrm{e}^{\psi}}{\xi^{2}} \frac{\mathrm{d} F}{\mathrm{~d} \xi}\right)+\frac{F(\xi)}{\xi^{2}}=0$

with $F(0)=0$ and

$\frac{\mathrm{d} F}{\mathrm{~d} \xi}+\frac{\mathrm{d} \psi}{\mathrm{d} \xi} F=0, \quad$ at $\quad \xi=\alpha$.

Now, the function $F(\xi)$ can be expressed as (Chavanis 2002a)

$F(\xi)=c_{1}\left(\xi^{3} \mathrm{e}^{-\psi}-\xi^{2} \psi^{\prime}\right)$.

Substituting this relation in the boundary condition (162), using the Emden Eq. (14) and introducing the Milne variables (16), we obtain the condition

$2 u_{0}-v_{0}=0$,

which coincides with the criterion (156) of thermodynamical stability. The expressions of the density perturbation profile and of the velocity profile at the point of marginal stability are given in Chavanis (2002a). Only the value of the critical point $\alpha$ changes. Due to the different boundary conditions, we now have $\delta v \neq 0$ at the surface of the gaseous sphere since the sphere can oscillate. The perturbation profile $\delta \rho / \rho$ has one node for the first mode of instability, two nodes for the second mode etc. The velocity profile $\delta v$ has no node for the first mode (except at the origin), one node for the second mode etc.

We could also consider the situation in which the pressure $P$ and the enthalpy $H=E+P V$ are fixed (instead of the temperature). This corresponds to the isenthalpic-isobaric ensemble (HBE). Since $\delta S=\frac{1}{T} \delta H-\frac{V}{T} \delta P$, the entropy must be a maximum at fixed $H$ and $P$ at statistical equilibrium. Eliminating the radius $R$ in Eqs. (19) and (20) in profit of the pressure $P$, we get after some algebra

$$
\begin{aligned}
\Lambda^{\prime} & \equiv \frac{-H}{(4 \pi)^{1 / 4} G^{3 / 4} M^{3 / 2} P^{1 / 4}}=\frac{1}{\left(u_{0} v_{0}^{3}\right)^{1 / 4}}\left(\frac{3}{2}-\frac{4}{3} u_{0}\right), \\
\eta^{\prime} & \equiv(4 \pi)^{1 / 4} G^{3 / 4} M^{1 / 2} P^{1 / 4} \beta=\left(u_{0} v_{0}^{3}\right)^{1 / 4} .
\end{aligned}
$$




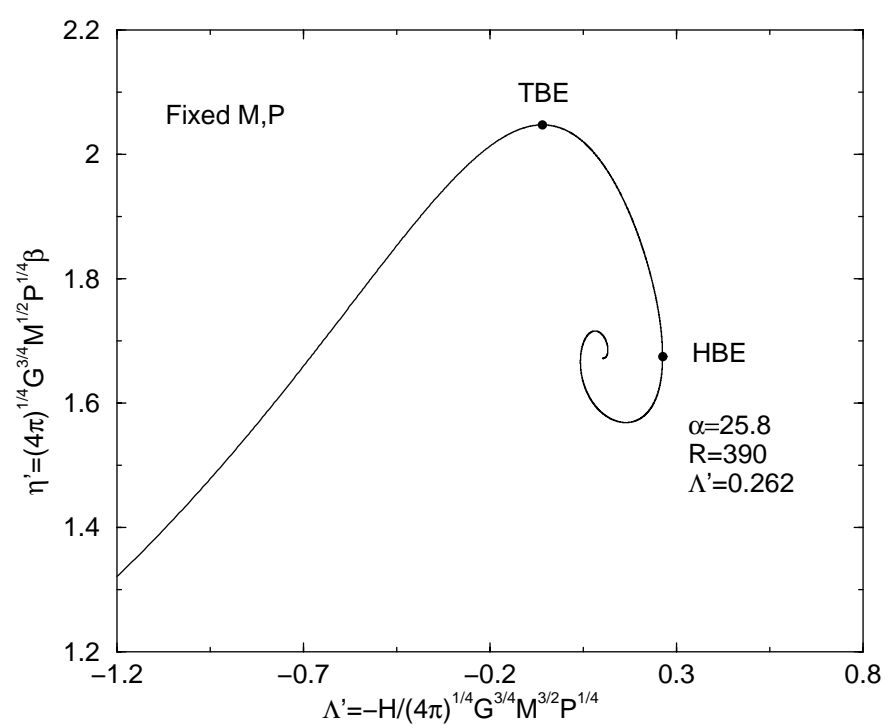

Fig. 17. Temperature vs. enthalpy plot at fixed pressure and mass. The series of equilibria becomes unstable at $\mathrm{HBE}$ in the isenthalpicisobaric ensemble.

The $T-H$ curve in HBE is plotted in Fig. 17. It spirals around the fixed point $\left(\Lambda_{\mathrm{s}}^{\prime}, \eta_{\mathrm{s}}^{\prime}\right)=\left(2^{1 / 4} / 12,2^{3 / 4}\right)$ corresponding to the singular solution. There is no equilibrium state for $\Lambda^{\prime}>0.262$. This implies a minimum enthalpy for a fixed pressure or a maximum pressure for a fixed enthalpy. This is similar to the Antonov (1962) criterion, the pressure playing the same role as the box radius. The series of equilibria becomes unstable in HBE at the point of minimum enthalpy. The condition $\mathrm{d} \Lambda^{\prime} / \mathrm{d} \alpha=0$ gives

$16 u_{0}^{2}+8 u_{0} v_{0}-38 u_{0}+3 v_{0}=0$.

The critical value of $\alpha$ is 25.8 corresponding to a density contrast of 390.

The specific heat at fixed pressure $C_{\mathrm{P}}=(\partial H / \partial T)_{N, P}$ can be deduced from Eqs. (165) and (166). After simplification, we find

$C_{\mathrm{P}}=\frac{1}{2} N k \frac{16 u_{0}^{2}+8 u_{0} v_{0}-38 u_{0}+3 v_{0}}{2 u_{0}-v_{0}}$.

For the ideal gas without gravity $\left(u_{0}, v_{0}\right)=(3,0)$ we recover the well-known result $C_{P}=\frac{5}{2} N k$. Gravitational instability occurs in the isenthalpic-isobaric ensemble when $C_{P}=0$ passing from negative to positive values and in the isothermal-isobaric ensemble when $C_{P}<0$.

\subsection{Polytropic gas spheres under external pressure}

We now consider a polytropic gas sphere of index $n$ (or $\gamma=1+$ $1 / n$ ) and mass $M$ under pressure $P$. Using Eqs. (96) and (102), the pressure on the boundary of the sphere is given by

$P=K \rho(R)^{\gamma}=K \rho_{0}^{1+\frac{1}{n}} \theta(\alpha)^{n+1}$.

The central density $\rho_{0}$ can be eliminated via the relation (104). Eliminating the radius $R$ via the relation (110) and introducing the Milne variables (105), we obtain after simplification

$\mathcal{P} \equiv \frac{1}{K}\left[\frac{4 \pi G^{3} M^{2}}{K^{3}(n+1)^{3}}\right]^{\frac{n+1}{n-3}} P=\left(u_{0} v_{0}^{3}\right)^{\frac{n+1}{n-3}}$.

Similarly, the normalized volume of the sphere is given by

$\mathcal{V} \equiv 3 V\left[\frac{K^{3}(1+n)^{3}}{4 \pi G^{3} M^{\frac{3(n-1)}{n}}}\right]^{\frac{n}{n-3}}=\frac{1}{\left(u_{0} v_{0}^{n}\right)^{\frac{3}{n-3}}}$.

Using the expansion of the Milne variables for $\alpha \rightarrow 0$,

$u_{0}=3-\frac{n}{5} \alpha^{2}+\ldots, \quad v_{0}=\frac{1}{3} \alpha^{2}-\ldots$

we find that the first order correction to the classical adiabatic law due to self-gravity is

$P V^{\gamma}=K M^{\gamma}-\lambda \frac{G M^{2}}{V^{\frac{n-3}{3 n}}}$,

Equation (173) can also be obtained from the Virial theorem (155) as before. The $P-V$ curve is plotted in Figs. 18 and 19 for different values of the polytropic index $n$. The volume is extremum at points $\alpha$ such that $\mathrm{d} \mathcal{V} / \mathrm{d} \alpha=0$. This leads to the condition $u_{0}=u_{\mathrm{s}}$. The pressure is extremum at points $\alpha$ such that $\mathrm{d} \mathcal{P} / \mathrm{d} \alpha=0$. This leads to the condition

$2 u_{0}+(3-n) v_{0}=0$.

The number of extrema can be obtained by a graphical construction in the $(u, v)$ plane like in Chavanis (2002b). For $n<3$, there is no extremum for $\mathcal{P}$ and $\mathcal{V}$. The pressure is a decreasing function of the volume and vanishes for a maximum volume $V_{\max }$ corresponding to the complete polytrope (such that $\left.\alpha=\xi_{1}\right)$. For $n=1$, the solution of the Lane-Emden equation is $\theta=\sin \xi / \xi$ for $\xi \leq \xi_{1}=\pi$ and we explicitly obtain

$\mathcal{P}=\frac{\sin ^{2} \alpha}{\alpha^{2}(\sin \alpha-\alpha \cos \alpha)^{2}}, \quad \mathcal{V}=\alpha^{3}$,

for $\alpha \leq \pi$. For $3<n \leq 5$, there is one extremum for $\mathcal{P}$ and $\mathcal{V}$. This implies the existence of a maximum pressure $P_{\max }$ and a minimum volume $V_{\min }$. In particular, for $n=5$, the LaneEmden equation can be solved analytically and we get

$\mathcal{P}=\frac{\alpha^{18}}{3^{6}\left(1+\frac{1}{3} \alpha^{2}\right)^{12}}, \quad \mathcal{V}=\frac{3^{6}}{\alpha^{15}}\left(1+\frac{1}{3} \alpha^{2}\right)^{9}$.

The pressure is maximum for $\alpha=3$ and the volume is minimum for $\alpha=\sqrt{15}$. For $n>5$ there is an infinity of extrema for $\mathcal{P}$ and $\mathcal{V}$. The $P-V$ curve spirals towards the limit point $\left(\mathcal{V}_{\mathrm{s}}, \mathcal{P}_{\mathrm{s}}\right)$ corresponding to the singular sphere. From turning point arguments, the series of equilibria becomes thermodynamically unstable at the point of maximum pressure (for $n>3$ ). Polytropes with $n<3$ are always stable in the isobaric ensemble.

Using Eqs. (170), (171) and the identities (106) and (107), we readily obtain

$\frac{\mathrm{d} \ln \mathcal{V}}{\mathrm{d} \alpha}=-\frac{3}{\alpha}\left(\frac{n-1}{n-3} u_{0}-1\right)$ 


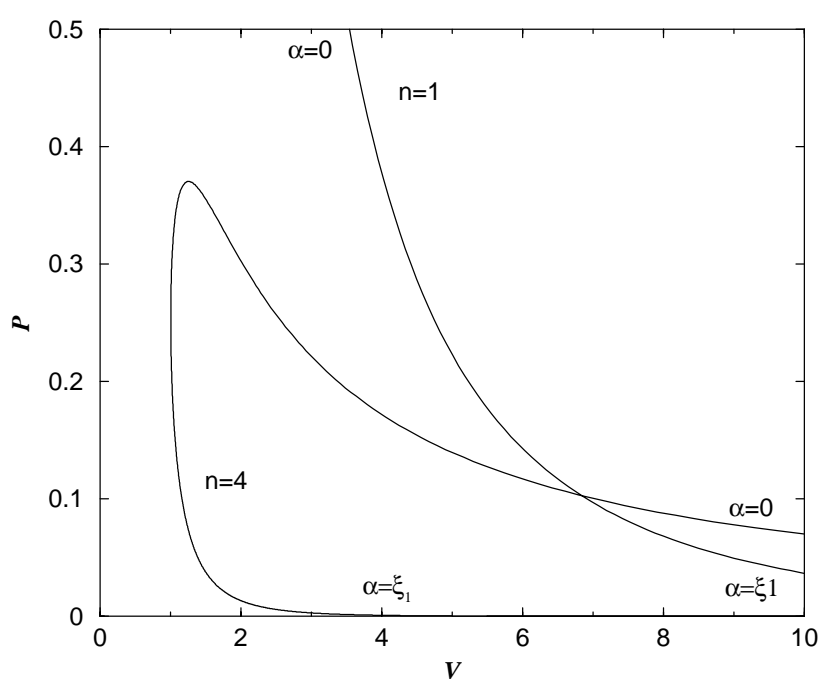

Fig. 18. The $P-V$ diagram for polytropes with $1 \leq n<3$ (specifically $n=1$ ) and $3<n \leq 5$ (specifically $n=4$ ).

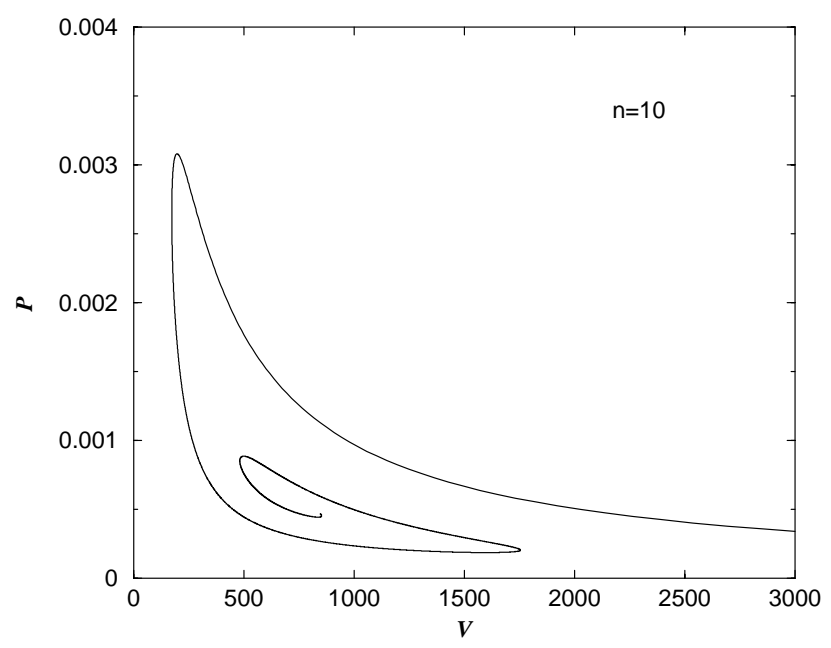

Fig. 19. The $P-V$ diagram for polytropes with $n>5$ (specifically $n=10)$.

and

$\frac{\mathrm{d} \ln \mathcal{P}}{\mathrm{d} \alpha}=\frac{n+1}{(n-3) \alpha}\left((3-n) v_{0}+2 u_{0}\right)$.

Therefore, the "compressibility" $\kappa=(\partial \ln P / \partial \ln V)_{N, K}$ can be expressed as

$\kappa=-\frac{1}{3}(n+1) \frac{2 u_{0}+(3-n) v_{0}}{(n-1) u_{0}-n+3}$.

We now consider the dynamical stability of polytropic gas spheres under external pressure. The equation of radial pulsations for a gas with a polytropic equation of state can be written (Chavanis 2002b)

$K \gamma \frac{\mathrm{d}}{\mathrm{d} r}\left(\frac{\rho^{\gamma-2}}{4 \pi r^{2}} \frac{\mathrm{d} q}{\mathrm{~d} r}\right)+\frac{G q}{r^{2}}=\frac{\lambda^{2}}{4 \pi \rho r^{2}} q$.

In the isobaric ensemble, the boundary conditions are given by Eq. (C.4) of Appendix C. Introducing dimensionless variables and considering the case of marginal stability $\lambda=0$, we are led to solve the problem

$\frac{\mathrm{d}}{\mathrm{d} \xi}\left(\frac{\theta^{1-n}}{\xi^{2}} \frac{\mathrm{d} F}{\mathrm{~d} \xi}\right)+\frac{n F(\xi)}{\xi^{2}}=0$,

with $F(0)=0$ and

$\theta \frac{\mathrm{d} F}{\mathrm{~d} \xi}-n \frac{\mathrm{d} \theta}{\mathrm{d} \xi} F=0, \quad$ at $\quad \xi=\alpha$.

Now, the function $F(\xi)$ can be expressed as (Chavanis 2002b)

$F(\xi)=c_{1}\left(\xi^{3} \theta^{n}+\frac{n-3}{n-1} \xi^{2} \theta^{\prime}\right)$.

Substituting this relation in the boundary condition (182), using the Lane-Emden Eq. (103) and introducing the Milne variables (105), we obtain the condition

$2 u_{0}+(3-n) v_{0}=0$,

which coincides with the criterion (174) of thermodynamical stability. The expressions of the density perturbation profile and of the velocity profile at the point of marginal stability are given in Chavanis (2002b). Only the value of the critical point $\alpha$ changes. For $3<n \leq 5$, the density perturbation profile has one node and the velocity profile no node (except at the origin). For $n>5$, the perturbation profile $\delta \rho / \rho$ has one node for the first mode of instability, two nodes for the second mode etc. The velocity profile $\delta v$ has no node for the first mode (except at the origin), one node for the second mode etc.

\section{Stability of galaxies and stars}

In this section, we discuss in detail the relation between the dynamical and the thermodynamical stability of stellar systems and gaseous stars following our previous investigations (Chavanis 2002a, b). This discussion should clarify the notion of generalized thermodynamics introduced by Tsallis (1988).

\subsection{Collisional vs. collisionless relaxation}

Basically, a stellar system is a collection of $N$ stars in gravitational interaction. Due to the development of encounters between stars, this system is expected to relax towards a statistical equilibrium state. The relaxation time can be estimated by $t_{\text {relax }} \sim(N / \ln N) t_{\mathrm{D}}$ where $t_{\mathrm{D}}$ is the dynamical time, i.e. the time it takes a star to cross the system (Binney \& Tremaine 1987). For globular clusters, $N \sim 10^{5}, t_{\mathrm{D}} \sim 10^{5}$ years so that the relaxation time is of the same order as their age $\left(\sim 10^{10}\right.$ years). Therefore, globular clusters form a discret Hamiltonian system which can be studied by ordinary methods of statistical mechanics. In particular, it can be shown rigorously that the Boltzmann entropy $S_{\mathrm{B}}[f]$ is the correct form of entropy for these systems (in a suitable thermodynamic limit) although they are non-extensive and non-additive. The absence of entropy maximum in an unbounded domain simply reflects the natural tendency of a stellar system to evaporate under the effect of encounters. However, evaporation is a slow process so that a globular cluster passes by a succession of quasiequilibrium states corresponding to truncated isothermals (see 
Sect. 4). It should be emphasized that statistical mechanics is based on the postulate that the evolution is ergodic. Now, the kinetic theory of self-gravitating systems is not wellunderstood (Kandrup 1981). Indeed, it is possible to prove a $\mathrm{H}$-theorem for the Boltzmann entropy only if simplifying assumptions are implemented (Markovian approximation, local approximation, regularization of logarithmic divergences,...). It would be of interest to determine more precisely the influence of non-ideal effects on the relaxation of stars. However, these effects are not expected to induce a strong deviation to Boltzmann's law. In particular, the diffusion of stars is normal or slightly anomalous, i.e. polluted by logarithmic corrections (Lee 1968). Hence, Tsallis entropies, which often arise in the case of anomalous diffusion, do not seem to be justified in the context of "collisional" stellar systems. However, there are many effects that can induce a lack of ergodicity and a deviation to Boltzmann's law. To a large extent, the dynamics of self-gravitating systems remains misunderstood and additional work (using numerical simulations) is necessary to clarify the process of collisional relaxation.

For elliptical galaxies, on the other hand, the relaxation time due to close encounters is larger than their age by about ten orders of magnitude $\left(N \sim 10^{11}, t_{\mathrm{D}} \sim 10^{8}\right.$ years, age $\sim 10^{10}$ years). Therefore, their dynamics is essentially encounterless and described by the self-consistent VlasovPoisson system. Elliptical galaxies form therefore a continuous Hamiltonian system. Now, due to a complicated mixing process in phase space, the Vlasov-Poisson system can achieve a metaequilibrium state (on a coarse-grained scale) on a very short timescale. A statistical theory appropriate to this violent relaxation was developed by Lynden-Bell in 1967 (see a short review in Chavanis 2002f). For collisionless stellar systems, the infinite mass problem is solved by realizing that violent relaxation is incomplete so that the galaxies are more confined that one would expect on the basis of statistical considerations.

Collisionless and collisional relaxations are therefore two distinct processes in the life of a self-gravitating system and they must be studied by different types of statistical mechanics. Note that a globular cluster first experiences a collisionless relaxation leading to a virialized state and then evolves more slowly towards another equilibrium state due to stellar encounters. We can discuss these two successive equilibrium states in a more formal manner. Let us consider a fixed interval of time $t$ and let the number of particles $N \rightarrow+\infty$. In that case, the system is rigorously described by the Vlasov equation, and a metaequilibrium state is achieved on a timescale independant on $N$ as a result of violent relaxation. Alternatively, if we fix $N$ and let $t \rightarrow+\infty$, the system is expected to relax to an equilibrium state resulting from a collisional evolution. These two equilibrium states are of course physically distinct. This implies that the order of the limits $N \rightarrow+\infty$ and $t \rightarrow+\infty$ is not interchangeable for self-gravitating systems (Chavanis 2002e).

\subsection{The Vlasov-Poisson system}

In Sects. 2-4, we have implicitly considered the case of collisional stellar systems, such as globular clusters, relaxing via two-body encounters. We now turn to the case of collisionless stellar systems, such as elliptical galaxies, described by the Vlasov equation

$$
\frac{\partial f}{\partial t}+\boldsymbol{v} \frac{\partial f}{\partial \boldsymbol{r}}+\boldsymbol{F} \frac{\partial f}{\partial \boldsymbol{v}}=0
$$

coupled with the Poisson Eq. (3). The Vlasov Eq. (185) simply states that, in the absence of encounters, the distribution function $f$ is conserved by the flow in phase space. This equation conserves the usual invariants (1) and (2) but also an additional class of invariants called the Casimirs

$I_{h}=\int h(f) \mathrm{d}^{3} \boldsymbol{r} \mathrm{d}^{3} \boldsymbol{v}$,

where $h$ is any continuous function of $f$. This infinite set of additional constraints is due to the collisionless nature of the evolution and results from the conservation of $f$ and the incompressibility of the flow in phase space. As we shall see, they play a crucial role in the statistical mechanics of violent relaxation. We note that the conservation of the Casimirs is equivalent to the conservation of the moments

$M_{n}=\int f^{n} \mathrm{~d}^{3} \boldsymbol{r} \mathrm{d}^{3} \boldsymbol{v}$

It is well-known that any distribution function of the form $f=f(\epsilon)$, where $\epsilon=\frac{v^{2}}{2}+\Phi$ is the individual energy of a star, is a stationary solution of the Vlasov equation. This is a particular case of the Jeans theorem (Binney \& Tremaine 1987). We shall assume furthermore that $f^{\prime}(\epsilon)<0$ so that high energy stars are less probable than low energy stars. Any such distribution function can be obtained by extremizing a functional of the form

$S[f]=-\int C(f) \mathrm{d}^{3} \boldsymbol{r} \mathrm{d}^{3} \boldsymbol{v}$,

at fixed mass $M$ and energy $E$, where $C(f)$ is a convex function, i.e. $C^{\prime \prime}(f) \geq 0$. Furthermore, maxima of $S[f]$ at fixed mass and energy are dynamically stable with respect to the VlasovPoisson system (Ipser 1974; Ipser \& Horwitz 1979). This is a sufficient condition of nonlinear stability. We are tempted to believe that it is also a necessary condition of nonlinear stability although this seems to go against the theorem of Doremus et al. (1971) which states that all distribution functions with $f^{\prime}(\epsilon)<0$ are dynamically stable (even if they are minima or saddle points of $S$ at fixed $E$ and $M$ ). In fact, this criterion is only a condition of linear stability (Binney \& Tremaine 1987) and it may not be valid for box confined models (its general applicability has also been criticized). In the following, we shall consider that $f=f(\epsilon)$ is dynamically stable "in a strong sense" if and only if it is a maximum of $S[f]$ at fixed $E$ and $M$. This is equivalent to $f=f(\epsilon)$ being a minimum of $E$ at fixed $S$ and $M$ (Ipser \& Horwitz 1979). It can be shown that stellar systems satisfying this requirement are necessarily spherically symmetric.

Introducing appropriate Lagrange multipliers and writing the variational principle in the form

$\delta S-\beta \delta E-\alpha \delta M=0$, 
one finds that

$C^{\prime}(f)=-\beta \epsilon-\alpha$.

Since $C^{\prime}$ is a monotonically increasing function of $f$, we can inverse this relation to obtain

$f=F(\beta \epsilon+\alpha)$,

where $F(x)=\left(C^{\prime}\right)^{-1}(-x)$. From the identity

$f^{\prime}(\epsilon)=-\beta / C^{\prime \prime}(f)$,

resulting from Eq. (190), $f(\epsilon)$ is a monotonically decreasing function of energy if $\beta>0$.

We note also that for each stellar systems with $f=f(\epsilon)$, there exist a corresponding barotropic gas with the same equilibrium density distribution. Indeed $\rho=\int f \mathrm{~d}^{3} \boldsymbol{v}=\rho(\Phi)$, $p=\frac{1}{3} \int f v^{2} \mathrm{~d}^{3} \boldsymbol{v}=p(\Phi)$, hence $p=p(\rho)$. Writing explicitly the pressure in the form

$p=\frac{1}{3} \int_{\Phi}^{+\infty} F(\beta \epsilon+\alpha) 4 \pi[2(\epsilon-\Phi)]^{3 / 2} \mathrm{~d} \epsilon$,

and taking its gradient, we obtain the condition of hydrostatic equilibrium

$\nabla p=-\rho \nabla \Phi$.

Therefore, the Jeans equations of stellar dynamics reduce in these cases of spherical systems with isotropic pressure to the equation of hydrostatics for a barotropic gas.

\subsection{Violent relaxation of collisionless stellar systems}

The Vlasov equation does not select a universal form of entropy $S[f]$, unlike the Boltzmann equation for example. Therefore, the notion of equilibrium is relatively subtle. When coupled to the Poisson equation, the Vlasov equation develops very complex filaments as a result of a mixing process in phase space. In this sense, the fine-grained distribution function $f(\boldsymbol{r}, \boldsymbol{v}, t)$ will never converge towards a stationary solution $f(\boldsymbol{r}, \boldsymbol{v})$. This is consistent with the fact that the functionals $S[f]$ are rigorously conserved by the flow (they are particular Casimirs). However, if we introduce a coarse-graining procedure, the coarse-grained distribution function $\bar{f}(\boldsymbol{r}, \boldsymbol{v}, t)$ will relax towards a metaequilibrium state $\bar{f}(\boldsymbol{r}, \boldsymbol{v})$. This collisionless relaxation is called $v i$ olent relaxation (Lynden-Bell 1967) or chaotic mixing. It can be shown that $S[\bar{f}]$ calculated with the coarse-grained distribution function at time $t>0$ is larger than $S[f]$ calculated with the fine-grained distribution function at $t=0$ (a monotonic increase of $H$ is not implied). This is similar to the $H$-theorem in kinetic theory except that $S[f]$ is not necessarily the Boltzmann entropy. For that reason, $S[f]$ is sometimes called a $H$-function (Tremaine et al. 1986). Boltzmann and Tsallis functionals $S_{\mathrm{B}}[f]$ and $S_{\mathrm{q}}[f]$ are particular $H$-functions corresponding to isothermal stellar systems and stellar polytropes.

It is important to note, furthermore, that among the infinite set of integrals conserved by the Vlasov equation, some are more robust than others. For example, the moments $M_{n}^{\text {c.g. }}[\bar{f}]=\int \bar{f}^{n} \mathrm{~d}^{3} \boldsymbol{r} \mathrm{d}^{3} \boldsymbol{v}$ calculated with the coarse-grained distribution function vary with time for $n>1$ since $\overline{f^{n}} \neq \bar{f}^{n}$.
By contrast, the mass and the energy are approximately conserved on the coarse-grained scale. We shall therefore classify the collisionless invariants in two groups: the energy $E$ and the mass $M$ will be called robust integrals since they are conserved by the coarse-grained dynamics while the moments $M_{n}$ with $n>1$ will be called fragile integrals since they vary under the operation of coarse-graining. Note that, in practice, the moments $M_{n>1}$ are also altered at the fine-grained scale because, intrinsically, the system is not continuous but made of stars. Therefore, the graininess of the medium can break the conservation of some moments, in general those of high order, even if we are in a regime where the evolution is essentially encounterless.

\subsection{Lynden-Bell's theory of violent relaxation}

Now, the question of fundamental interest is the following: given some initial condition, can we predict the equilibrium distribution function that the system will eventually achieve? Lynden-Bell (1967) tried to answer this question by using statistical mechanics arguments. He introduced the density probability $\rho(\boldsymbol{r}, \mathbf{v}, \eta)$ of finding the value of the distribution function $\eta$ in $(\boldsymbol{r}, \boldsymbol{v})$ at equilibrium. Then, using a combinatorial analysis he showed that the relevant measure is the Boltzmann entropy

$S[\rho]=-\int \rho \ln \rho \mathrm{d}^{3} \boldsymbol{r d}^{3} \boldsymbol{v} d \eta$,

which is a functional of $\rho$. This mixing entropy has been justified rigorously by Michel \& Robert (1994), using the concept of Young measures. Therefore, although the system is non-extensive, the Boltzmann entropy is the correct measure. Assuming ergodicity (which may not be realized in practice, see Sect. 7.5) the most probable equilibrium state, i.e. most mixed state, is obtained by maximizing $S[\rho]$ taking into account all the constraints of the dynamics. The optimal equilibrium state can be written

$\rho(\boldsymbol{r}, \boldsymbol{v}, \eta)=\frac{1}{Z} \chi(\eta) \mathrm{e}^{-(\beta \epsilon+\alpha) \eta}$,

where $\chi(\eta) \equiv \exp \left(-\sum_{n>1} \alpha_{n} \eta^{n}\right)$ accounts for the conservation of the fragile moments $M_{n>1}=\int \rho \eta^{n} \mathrm{~d}_{n} \mathrm{~d}^{3} \boldsymbol{r} \mathrm{d}^{3} \boldsymbol{v}$ and $\alpha, \beta$ are the usual Lagrange multipliers for $M$ and $E$ (robust integrals). The distinction between these two types of constraints is important (see below) and was not explicitly made by Lynden-Bell (1967). The "partition function"

$Z=\int_{0}^{+\infty} \chi(\eta) \mathrm{e}^{-(\beta \epsilon+\alpha) \eta} \mathrm{d} \eta$

is determined by the local normalization condition $\int \rho \mathrm{d} \eta=1$. The condition of thermodynamical stability reads

$\delta^{2} J \equiv-\frac{1}{2} \int \frac{(\delta \rho)^{2}}{\rho} \mathrm{d}^{3} \boldsymbol{r} \mathrm{d}^{3} \boldsymbol{v} \mathrm{d} \eta-\frac{1}{2} \beta \int \delta \Phi \delta \bar{f} \mathrm{~d}^{3} \boldsymbol{r} \mathrm{d}^{3} \boldsymbol{v} \leq 0$,

for all variation $\delta \rho(\boldsymbol{r}, \boldsymbol{v}, \eta)$ which does not change the constraints to first order. The equilibrium coarse-grained distribution function $\bar{f} \equiv \int \rho \eta \mathrm{d} \eta$ can be expressed as

$\bar{f}=-\frac{1}{\beta} \frac{\partial \ln Z}{\partial \epsilon}=F(\beta \epsilon+\alpha)=f(\epsilon)$. 
Taking the derivative of Eq. (199), it is easy to show that

$$
\bar{f}^{\prime}(\epsilon)=-\beta f_{2}, \quad f_{2} \equiv \int \rho(\eta-\bar{f})^{2} \mathrm{~d} \eta>0,
$$

where $f_{2}$ is the centered local variance of the distribution $\rho(\boldsymbol{r}, \boldsymbol{v}, \eta)$. The integrability condition $\int f \mathrm{~d}^{3} \boldsymbol{v}<\infty$ requires that the temperature is positive $(\beta>0)$. Then, from Eq. (200), we deduce that $f$ is a monotonically decreasing function of $\epsilon$. Therefore, from Sect. 7.2, we know that for each function $f(\epsilon)$ of the form (199) there exists a $H$-function $S[\bar{f}]$ whose extremization at fixed mass and energy returns Eq. (199). Furthermore, it can be shown that the condition (198) of thermodynamical stability (maximum of $S[\rho]$ at fixed $E$ and $M_{n}$ ) implies that $\bar{f}$ is a nonlinearly stable solution of the VlasovPoisson system. Therefore, it is a maximum of $S[\bar{f}]$ at fixed mass and energy. This functional depends on the initial conditions through the Lagrange multipliers $\alpha_{n>1}$, or equivalently through the function $\chi(\eta)$, which are determined by the fragile moments $M_{n>1}$. Therefore, $S[\bar{f}]$ is non-universal and can take a wide variety of forms. In general, $S[\bar{f}]$ differs from the ordinary Boltzmann entropy $S_{\mathrm{B}}[\bar{f}]$ derived in the context of collisional stellar systems. This is due to the additional constraints (Casimir invariants) brought by the Vlasov equation. For a given initial condition, the $H$-function $S[\bar{f}]$ maximized by the system at statistical equilibrium is uniquely determined by the statistical theory according to Eqs. (190) and (199).

In general, $S[\bar{f}]$ is not an entropy, unlike $S[\rho]$, as it cannot be directly obtained from a combinatorial analysis. There is a case, however, where $S[\bar{f}]$ is an entropy. If the initial condition is made of patches with value $f_{0}=\eta_{0}$ and $f_{0}=0$ (two-levels approximation), then the entropy $S[\rho]$ is equivalent to the Fermi-Dirac entropy $S_{\text {F.D. }}[\bar{f}]=-\int\left[\bar{f} / \eta_{0} \ln \bar{f} / \eta_{0}+\right.$ $\left.\left(1-\bar{f} / \eta_{0}\right) \ln \left(1-\bar{f} / \eta_{0}\right)\right] \mathrm{d}^{3} \boldsymbol{r} \mathrm{d}^{3} \boldsymbol{v}$ and Lynden-Bell's distribution (199) coincides with the Fermi-Dirac distribution (LyndenBell 1967; Chavanis \& Sommeria 1998). Moreover, all the moments can be expressed in terms of the mass alone as $M_{n}=\eta_{0}^{n} M$. In the non-degenerate (or dilute) limit $\bar{f} \ll \eta_{0}$, the Fermi-Dirac entropy reduces to the Boltzmann entropy $S_{\mathrm{B}}[\bar{f}]=-\int \bar{f} \ln \bar{f} \mathrm{~d}^{3} \boldsymbol{r} \mathrm{d}^{3} \boldsymbol{v}$. Quite generally, in the dilute limit, the equilibrium distribution is a sum of isothermal distributions.

We can note some general predictions of the statistical theory, which are valid for any initial condition $f_{0}(\boldsymbol{r}, \boldsymbol{v})$. (i) The predicted distribution function is a function $\bar{f}=f(\epsilon)$ of the stellar energy alone in the non-rotating case and a function $\bar{f}=f\left(\epsilon_{\mathrm{J}}\right)$ of the Jacobi energy $\epsilon_{\mathrm{J}}=\epsilon-\boldsymbol{\Omega} \cdot(\boldsymbol{r} \times \boldsymbol{v})$ alone in the rotating case. (ii) $f^{\prime}(\epsilon)<0$ so that high energy stars are less probable than low energy stars. (iii) $\bar{f}(\epsilon)$ is strictly positive for all energies (it never vanishes). (iv) $\bar{f}(\epsilon)$ is always smaller than the maximum value $f_{0}^{\max }$ of the initial condition. (v) According to Eqs. (200) and (192), we have the general relation $f_{2}=1 / C^{\prime \prime}(\bar{f})$. (vi) For a box-confined system, there always exist a global maximum of entropy. Therefore, contrary to collisional stellar systems, it is not necessary to introduce a small-scale cut-off to make the thermodynamical approach rigorous. This is due to the Liouville theorem which puts an upper bound on the value of the distribution function like the
Pauli exclusion principle in quantum mechanics (see Chavanis et al. 1996; Chavanis \& Sommeria 1998).

\subsection{Incomplete relaxation}

The prediction of Lynden-Bell's statistical theory crucially depends on the initial conditions. This is because we need to know the value of the moments $M_{n>1}$ which can only be deduced from the fine-grained distribution function at $t=0$ (say). Indeed, these moments are fragile, or microscopic, constraints which cannot be mesured from the coarse-grained flow at $t>0$ since they are altered by the coarse-graining procedure. This is a specificity of continuous Hamiltonian systems which contrasts with ordinary (discrete) Hamiltonian systems for which the constraints are robust, or macroscopic, and can be evaluated at any time (as the mass $M$ and the energy $E$ ). Now, Lynden-Bell (1967) gives arguments according to which elliptical galaxies should be non-degenerate (except possibly in the nucleus). In that case, the dilute limit of his theory applies in the main body of the galaxy and leads to an isothermal distribution. The theoretical justification of an isothermal distribution for collisionless stellar systems was considered as a triumph in the 1960's. Indeed, Lynden-Bell's statistical theory of violent relaxation could explain the observed isothermal core of elliptical galaxies without recourse to collisions that operate on a much longer timescale.

However, it is easy to see that the statistical theory of violent relaxation cannot describe the halo of elliptical galaxies. Indeed, at large distances, the density decreases as $r^{-2}$ resulting in the infinite mass problem. Lynden-Bell (1967) has related this mathematical difficulty to the physical problem of incomplete relaxation and argued that his distribution function has to be modified at high energies. Indeed, the relaxation is effective only in a finite region of space and persists only for a finite period of time. Therefore, there is no reason to maximize entropy in the whole available space. The ergodic hypothesis which sustains the statistical theory applies only in a restricted domain of space, in a sort of "maximum entropy bubble", surrounded by an unmixed region which is only poorly sampled by the system. Accordingly, the physical picture that emerges is the following: during violent relaxation, the system has the tendency to reach the most mixed state described by the distribution (199). However, as it approaches equilibrium, the mixing becomes less and less efficient and the system settles on a state which is not the most mixed state.

How can we take into account incomplete relaxation? A first possibility is to confine the system artificially inside a box, where the box delimits the typical region in which the statistical theory applies (Chavanis \& Sommeria 1998). Another possibility is to use a parametrization of the coarse-grained dynamics of collisionless stellar systems in the form of relaxation equations involving a space and time dependant diffusion coefficient related to the fine-grained fluctuations of the distribution function (Chavanis et al. 1996; Chavanis 1998). This space and time dependant diffusion coefficient can freeze the system in a "maximum entropy bubble" and lead to the formation of self-confined stellar systems. Finally, a third possibility is to 
construct a model of incomplete violent relaxation which provides a depletion of stars in the high energy tail of the distribution function. If the system is isolated, as most elliptical galaxies are, Hjorth \& Madsen (1993) have proposed a dynamical scenario attempting to take into account incomplete relaxation in the halo. They introduce a two-step process: (i) in a first step, they assume that violent relaxation proceeds to completion in a finite spatial region, of radius $r_{\max }$, which represents roughly the core of the galaxy (where the fluctuations are important). At this stage, the escape energy represents no special threshold so that negative as well as positive energy states are populated in that region. (ii) After the relaxation process is over, positive energy particles leave the system and particles with $\Phi\left(r_{\max }\right)<\epsilon<0$ move in orbits beyond $r_{\max }$, thereby changing the distribution function to something significantly "thinner" than a Boltzmann distribution. The crucial point to realize is that the differential energy distribution $N(\epsilon)$, where $N(\epsilon) \mathrm{d} \epsilon$ is the number of stars with energy between $\epsilon$ and $\epsilon+\mathrm{d} \epsilon$, will be discontinuous at the escape energy $\epsilon=0$ since there is a finite number of particles within $r_{\max }$ after the relaxation process. It can be shown that this discontinuity implies necessarily that $f(\epsilon) \sim(-\epsilon)^{5 / 2}$ for $\epsilon \rightarrow 0^{-}$(Jaffe 1987). Therefore, the distribution function consistent with this scenario is (Hjorth \& Madsen 1993)

$\bar{f}=\left\{\begin{array}{cc}A \mathrm{e}^{-\beta \eta_{0} \epsilon} & \Phi_{0} \leq \epsilon<\epsilon^{\prime}, \\ B(-\epsilon)^{5 / 2} & \epsilon^{\prime} \leq \epsilon<0, \\ 0 & \epsilon \geq 0 .\end{array}\right.$

This model corresponds formally to a composite configuration with an isothermal core in which violent relaxation is efficient and a polytropic halo (with index $n=4$ ) which is only partially relaxed (in reality, there is a small deviation to the ideal value $n=4$ because the potential created by the core is not exactly Keplerian; see Hjorth \& Madsen 1993). For this model, the density decreases as $r^{-4}$ like in elliptical galaxies. Furthermore, this model can reproduce de Vaucouleur's $R^{1 / 4}$ law for the surface brightness of ellipticals (Binney \& Tremaine 1987). Alternatively, if the system is subject to tidal forces (this may be the case for dark matter halos), one can propose an extended Michie-King model of the form (Chavanis 1998)

$\bar{f}=\left\{\begin{array}{cl}\eta_{0} \frac{\mathrm{e}^{-\beta \eta_{0} \epsilon}-\mathrm{e}^{-\beta \eta_{0} \epsilon m}}{\lambda+\mathrm{e}^{-\beta \eta_{0} \epsilon}} & \epsilon<\epsilon_{m}, \\ 0 & \epsilon \geq \epsilon_{m},\end{array}\right.$

which takes into account the specificities of Lynden-Bell's distribution function (in particular the exclusion principle associated with Liouville's theorem) and leads to a confinement of the density at large distances (i.e. the density drops to zero at a finite distance identified with the tidal radius).

\subsection{Tsallis entropies}

The statistical theory of Lynden-Bell encounters some problems because the distribution function $f(\epsilon)$ never vanishes. Therefore, we have to invoke incomplete relaxation and introduce dynamical constraints in order to explain the observation of confined galaxies and solve the infinite mass problem. This is probably the correct approach to the problem. Another line of thought, defended by Tsallis and co-workers, is to change the form of entropy in order to obtain a distribution function that vanishes at some maximum energy. Therefore, Tsallis generalized thermodynamics is essentially an attempt to take into account incomplete mixing and lack of ergodicity in complicated systems. If we assume that the system is classically described by the Boltzmann entropy $S_{\mathrm{B}}[f]$ (which is true for collisionless stellar systems only in a coarse-grained sense and in a dilute limit), then Tsallis proposes to replace $S_{\mathrm{B}}[f]$ by a $q$-entropy $S_{q}[f]$. The parameter $q$ measures the efficiency of mixing ( $q=1$ if the system mixes well) but is not determined by the theory.

This approach is attractive but the justification given by Tsallis and co-workers is misleading. Fundamentally, ordinary thermodynamics (in the sense of Lynden-Bell ) is the correct approach to the problem. The Boltzmann entropy $S[\rho]$ has a clear physical meaning as it is proportional to the logarithm of the disorder, where the disorder is equal to the number of microstates consistent with a given macrostate. Of course, if the system does not mix well, statistical mechanics loses its power of prediction. However, the state resulting from incomplete violent relaxation is always a nonlinearly stable stationary solution of the Vlasov equation on a coarse-grained scale (i.e., for $\bar{f}$ ). Therefore, if $\bar{f}=f(\epsilon)$, it maximizes a $H$-function $S[\bar{f}]=-\int C(\bar{f}) \mathrm{d}^{3} \boldsymbol{r} \mathrm{d}^{3} \boldsymbol{v}$ at fixed mass $M$ and energy $E$. The function $C(f)$ is influenced by thermodynamics (mixing) but it cannot be determined by pure thermodynamical arguments because of incomplete relaxation.

The $H$-function $S[f]$ selected by the system depends both on the initial condition and on the type of mixing. Tsallis entropy $S_{q}[f]$ is just a particular $H$-function leading to confined structures (stellar polytropes). Now, it is well-known that stellar polytropes give a poor fit of elliptical galaxies. In particular, they cannot reproduce de Vaucouleur's law, neither the $r^{-4}$ density decrease of these objects. Indeed, for stellar polytropes, the density decreases as $r^{-5}$ for $n=5$, the density drops to zero at a finite distance for $n<5$ and the mass is infinite for $n>5$. We conclude, therefore, that observations of elliptical galaxies do not support the prediction of Tsallis. The model of Hjorth \& Madsen (1993) based on the statistical theory of Lynden-Bell improved by a physically motivated scenario of incomplete relaxation provides a much better agreement with observations. This equilibrium state cannot be described by Tsallis distribution which fails to reproduce simultaneously an isothermal core and an external confinement. In fact, the model of Hjorth \& Madsen (1993) can be obtained by maximizing a $H$-function with

$$
C(f)=\left\{\begin{array}{cl}
f \ln f, & f>f_{0} \\
\frac{1}{q-1}\left(f^{q}-f\right), & f<f_{0},
\end{array}\right.
$$

at fixed mass and energy. Therefore, their model is consistent with a composite Tsallis model with $q=1$ (complete mixing) in the core and $q \equiv(2 n-1) /(2 n-3)=7 / 5$ (incomplete mixing) in the halo. More generally, we could argue that $q$ varies in space in order to take into account a variable mixing efficiency (very similarly, stars are sometimes approximated by composite polytropic models with a space dependent index in 
order to distinguish between convective and radiative regions). However, this gives to Tsallis distribution a practical, not a fundamental justification. It can just fit locally the distribution function of collisionless stellar systems resulting from incomplete violent relaxation.

In addition, not all stable stationary solutions of the Vlasov equation can be obtained by maximizing a $H$-function at fixed mass and energy. This maximization problem leads to $f=f(\epsilon)$ with $f^{\prime}(\epsilon)<0$. For spherical stellar systems, the distribution function can depend on the specific angular momentum $\mathbf{j}=\boldsymbol{r} \times \boldsymbol{v}$ in addition to the stellar energy $\epsilon$ and this is what happens in certain models of incomplete violent relaxation with anisotropic velocity distribution (Lynden-Bell 1967). On the other hand, for rotating stellar systems, the maximization of $S[f]$ at fixed mass, energy and angular momentum predicts that $f=f\left(\epsilon_{\mathrm{J}}\right)$ with $f^{\prime}<0$, where $\epsilon_{\mathrm{J}}$ is the Jacobi energy. However, it is well-known that such distribution function cannot account for the triaxial structure of elliptical galaxies. More general distribution functions must be constructed in agreement with the Jeans theorem (Binney \& Tremaine 1987). However, it is possible that relevant distribution functions maximize a $H$-function with additional constraints. These constraints may correspond to adiabatic invariants that are approximately conserved during violent relaxation. For example, anisotropic velocity distributions can be obtained by maximizing a $H$-function while conserving the individual distributions of angular momentum $\boldsymbol{L}_{2}=\int f(\boldsymbol{r} \times \boldsymbol{v})^{2} \mathrm{~d}^{3} \boldsymbol{r} \mathrm{d}^{3} \boldsymbol{v}$ (or other distribution) in addition to mass and energy. This leads to $f=f\left(\epsilon_{\mathrm{a}}\right)$ with $\epsilon_{\mathrm{a}} \equiv \epsilon+\frac{j^{2}}{2 r_{\mathrm{a}}}$ and $f^{\prime}\left(\epsilon_{\mathrm{a}}\right)<0\left(r_{\mathrm{a}}\right.$ is called the anisotropy radius $)$. It is expected that this maximization problem is a condition of nonlinear stability. For rotating systems, it is not known whether we can construct distribution functions yielding triaxial bodies by maximizing a $H$-function at fixed $M, E, \boldsymbol{L}$ and additional (adiabatic) invariants. These ideas may be a route to explore.

\subsection{Dynamical stability of collisionless stellar systems}

On a formal point of view, the maximization problem determining the nonlinear stability of a collisionless stellar system is similar to the one which determines the thermodynamical stability of a collisional stellar system. In thermodynamics, we maximize the Boltzmann entropy $S_{\mathrm{B}}[f]$ at fixed mass $M$ and energy $E$ in order to determine the most probable equilibrium state. In stellar dynamics, the maximization of a $H$-function $S[f]$ at fixed mass and energy determines a nonlinearly stable stationary solution of the Vlasov-Poisson system. Therefore, we can use a thermodynamical analogy to analyse the dynamical stability of collisionless stellar systems. In this analogy, the functional $S[f]$ plays the role of a generalized entropy, the Lagrange multiplier $\beta$ plays the role of a generalized inverse temperature $\beta=1 / T$, the curve $\beta(E)$ the role of a generalized caloric curve and the maximization of $S[f]$ at fixed $E$ and $M$ corresponds to a microcanonicial description. This analogy explains why we take $C$ to be convex (and not concave) in Eq. (188). Then, we must look for maxima of $S$ (not minima) like in thermodynamics. We can also introduce a $J$-function defined by $J[f] \equiv S-\beta E$ which is similar to a free energy in thermodynamics ( $J$ is the Legendre transform of $S$ ). The maximization of $J[f]$ at fixed $M$ and $\beta$ corresponds to a canonical description.

The condition that $f$ is a maximum of $S$ at fixed mass and energy is equivalent to the condition that $\delta^{2} J=\delta^{2} S-\beta \delta^{2} E$ is negative for all perturbations that conserve mass and energy to first order. This condition can be written

$$
\begin{array}{r}
\delta^{2} J=-\int C^{\prime \prime}(f) \frac{(\delta f)^{2}}{2} \mathrm{~d}^{3} \mathbf{r d}{ }^{3} \mathbf{v}-\frac{1}{2} \beta \int \delta \rho \delta \Phi \mathrm{d}^{3} \mathbf{r} \leq 0, \\
\forall \delta f \mid \delta E=\delta M=0 .
\end{array}
$$

The condition that $f$ is a maximum of $J=S-\beta E$ at fixed mass and temperature is equivalent to the condition that $\delta^{2} J$ is negative for all perturbations that conserve mass. This can be written

$$
\begin{array}{r}
\delta^{2} J=-\int C^{\prime \prime}(f) \frac{(\delta f)^{2}}{2} \mathrm{~d}^{3} \mathbf{r} \mathrm{d}^{3} \mathbf{v}-\frac{1}{2} \beta \int \delta \rho \delta \Phi \mathrm{d}^{3} \mathbf{r} \leq 0, \\
\forall \delta f \mid \delta M=0 .
\end{array}
$$

According to Eq. (192), $\delta^{2} J$ can be re-written

$\delta^{2} J=-\frac{1}{2} \beta\left\{\int \frac{(\delta f)^{2}}{-f^{\prime}(\epsilon)} \mathrm{d}^{3} \mathbf{r} \mathrm{d}^{3} \mathbf{v}+\int \delta \rho \delta \Phi \mathrm{d}^{3} \mathbf{r}\right\}$,

which is the usual form considered in the literature. We emphasize, however, that the role of the first order constraints is crucial in the stability analysis and is often underestimated. If $f$ is a maximum of $J$ at fixed $M$ and $\beta$ then it is necessarily a maximum of $S$ at fixed $M$ and $E$. Indeed, if the inequality (205) is satisfied for all perturbations that conserve mass, it is a fortiori satisfied for perturbations that conserve mass and energy. However, the reciprocal is wrong in general. In thermodynamics, this means that canonical stability implies microcanonical stability (but the converse is wrong in general). Exploiting this thermodynamical analogy, we can use the turning point arguments of Katz (1978) to settle whether a solution of the form (190) is a maximum or a minimum (or a saddle point) of $S$ or $J$. The canonical and microcanonical descriptions will be inequivalent if the curve $\beta(E)$ presents turning points leading to regions where $C=\mathrm{d} E / \mathrm{d} T<0$. This corresponds to regions of negative specific heats in thermodynamics. In these regions, the curve $S(E)$ has a convex dip. This convex dip is the signal of phase transitions in thermodynamics.

We can also try to reduce the stability problem to the study of an eigenvalue equation. To that purpose, it is convenient to first maximize $S[f]$ at fixed mass, energy and density $\rho(\mathbf{r})$ in order to obtain a functional $S[\rho]$. Now, maximizing $S[f]$ at fixed mass, energy and density is equivalent to maximizing $S[f]$ at fixed kinetic energy $K$ and density. Introducing a Lagrange multiplier $\beta$ for the kinetic energy and a space dependant Lagrange multiplier $\lambda(\boldsymbol{r})$ for the density constraint, we get

$f=F\left[\beta\left(\frac{v^{2}}{2}+\lambda(\boldsymbol{r})\right)\right]$,

where $F$ is the same function as in Sect. 7.2. Since

$\delta^{2} S=-\int C^{\prime \prime}(f) \frac{(\delta f)^{2}}{2} \mathrm{~d}^{3} \boldsymbol{r} \mathrm{d}^{3} \boldsymbol{v} \leq 0$, 
we conclude that Eq. (207) is a maximum of $S$ at fixed $K$ and $\rho$. This is also a maximum of $J=S-\beta E$ at fixed $\beta$ and $\rho$.

With the distribution function (207), we can write the density and the pressure as

$\rho=\frac{1}{\beta^{3 / 2}} g(\beta \lambda), \quad p=\frac{1}{\beta^{5 / 2}} h(\beta \lambda)$,

where

$g(x)=\int_{0}^{+\infty} 4 \pi \sqrt{2} F(t+x) t^{1 / 2} \mathrm{~d} t$,

and

$h(x)=\int_{0}^{+\infty} \frac{8 \pi \sqrt{2}}{3} F(t+x) t^{3 / 2} \mathrm{~d} t$.

We can now express $E$ and $S$ as functionals of $\rho$ and $\beta$. The energy (2) is simply given by

$E=\frac{3}{2} \int p d^{3} \boldsymbol{r}+\frac{1}{2} \int \rho \Phi \mathrm{d}^{3} \boldsymbol{r}$

On the other hand, according to Eq. (188), we have

$S=-4 \pi \sqrt{2} \int \mathrm{d}^{3} \boldsymbol{r} \int_{\lambda}^{+\infty} C[F(\beta \epsilon)](\epsilon-\lambda)^{1 / 2} \mathrm{~d} \epsilon$.

Setting $t=\beta(\epsilon-\lambda)$, we get

$S=-\frac{4 \pi \sqrt{2}}{\beta^{3 / 2}} \int \mathrm{d}^{3} \boldsymbol{r} \int_{0}^{+\infty} C[F(t+\beta \lambda)] t^{1 / 2} \mathrm{~d} t$.

Integrating by parts and using $C^{\prime}[F(x)]=-x$, we find that

$S=-\frac{8 \pi \sqrt{2}}{3 \beta^{3 / 2}} \int \mathrm{d}^{3} \boldsymbol{r} \int_{0}^{+\infty} F^{\prime}(t+\beta \lambda)(t+\beta \lambda) t^{3 / 2} \mathrm{~d} t$.

Integrating by parts one more time and using Eqs. (209)-(211), we finally obtain

$S=\frac{5}{2} \beta \int p \mathrm{~d}^{3} \boldsymbol{r}+\beta \int \lambda \rho \mathrm{d}^{3} \boldsymbol{r}$.

We now consider the maximization of $S[\rho]$ at fixed mass and energy. This is similar to a microcanonical situation in thermodynamics. In that case, $\beta$ is not fixed and its variation $\delta \beta$ is related to the change of density $\delta \rho$ via the energy constraint (212), using Eqs. (209)-(211). This implies in particular that the barotropic relation $p=p(\rho)$, valid at equilibrium, is not preserved when we consider variations around equilibrium. Indeed, eliminating $\beta \lambda$ in Eq. (209), we find that $p=\beta^{-5 / 2} H\left(\beta^{3 / 2} \rho\right)$ so that $\beta$ enters explicitly in the relation between $p$ and $\rho$. In the case of isothermal stellar systems and stellar polytropes described by Boltzmann and Tsallis entropies, it is possible to reduce the stability problem to the study of an eigenvalue equation (Padmanabhan 1989; Taruya \& Sakagami 2002a). However, it seem difficult to make this reduction in the general case.

\subsection{Dynamical stability of gaseous spheres}

We now consider the maximization of $J[\rho]=S[\rho]-\beta E[\rho]$ at fixed $\beta$ and $M$. This is similar to a canonical situation in thermodynamics. According to Eqs. (212) and (216), we have

$J=\beta \int p \mathrm{~d}^{3} \boldsymbol{r}+\beta \int \lambda \rho \mathrm{d}^{3} \boldsymbol{r}-\frac{1}{2} \beta \int \rho \Phi \mathrm{d}^{3} \boldsymbol{r}$.

Since $\beta$ is constant, the barotropic relation $p=p(\rho)$ remains valid around equilibrium. Using the relation $h^{\prime}(x)=-g(x)$ obtained from Eqs. (210) and (211) by a simple integration by parts, it is easy to check that Eq. (209) yields

$\lambda+\frac{p}{\rho}=-\int_{0}^{\rho} \frac{p\left(\rho^{\prime}\right)}{\rho^{\prime 2}} \mathrm{~d} \rho^{\prime}$.

Therefore, the functional (217) can be rewritten as

$J=-\beta \int \rho \int_{0}^{\rho} \frac{p\left(\rho^{\prime}\right)}{\rho^{\prime 2}} \mathrm{~d} \rho^{\prime} \mathrm{d}^{3} \boldsymbol{r}-\frac{1}{2} \beta \int \rho \Phi \mathrm{d}^{3} \boldsymbol{r}$.

If we cancel the first order variations of $J$ at fixed mass and $\beta$, we get $\lambda=\Phi+\alpha / \beta$ and we recover Eq. (191). On the other hand, the condition that Eq. (191) is a maximum of $J$ at fixed $M$ and $\beta$ requires that the second order variations of $J$ be negative. This leads to the inequality

$\delta^{2} J=-\beta \int \frac{p^{\prime}(\rho)}{2 \rho}(\delta \rho)^{2} \mathrm{~d}^{3} \boldsymbol{r}-\frac{1}{2} \beta \int \delta \rho \delta \Phi \mathrm{d}^{3} \boldsymbol{r} \leq 0$,

for all perturbations $\delta \rho$ that conserve mass. Using the condition of hydrostatic equilibrium, $\delta^{2} J$ can be re-written

$\delta^{2} J=-\frac{1}{2} \beta\left\{\int \frac{(\delta \rho)^{2}}{-\rho^{\prime}(\Phi)} \mathrm{d}^{3} \boldsymbol{r}+\int \delta \rho \delta \Phi \mathrm{d}^{3} \boldsymbol{r}\right\}$.

We note, in passing, the similarity with the pseudo-energy (or Arnol'd invariant) in two-dimensional hydrodynamics (see Chavanis 2002h). Therefore, Arnol'd theorems can be directly extended to (3D) self-gravitating bodies. In particular, in a bounded domain, the system is nonlinearly stable, for any type of perturbation, if $0 \leq-\rho^{\prime}(\Phi) \leq \lambda_{1} /(4 \pi G)$, where $\lambda_{1}$ is the smallest eigenvalue of $-\Delta$ (for isothermal spheres this corresponds to $\alpha \leq \pi$ and for polytropic spheres to $\alpha \leq \pi / \sqrt{n}$ where $\alpha$ is defined by Eqs. (15) and (104) respectively). Furthermore, we have already shown in the case of isothermal stellar systems and stellar polytropes that the stability condition (220) can be reduced to the study of an eigenvalue equation. This reduction can be generalized to any stellar system described by a $H$-function $S[f]$ of the form (188). Considering spherically symmetrical perturbations, introducing the variable $q(r)$ defined by Eq. (122) and repeating exactly the same steps as for isothermal and polytropic spheres (see Chavanis 2002a and Sect. 5.5), we end up on the eigenvalue problem

$\left[\frac{\mathrm{d}}{\mathrm{d} r}\left(\frac{p^{\prime}(\rho)}{4 \pi \rho r^{2}} \frac{\mathrm{d}}{\mathrm{d} r}\right)+\frac{G}{r^{2}}\right] q_{\lambda}(r)=\lambda q_{\lambda}(r)$,

with appropritate boundary conditions (see Appendix C). If all the $\lambda$ 's are negative, then Eq. (191) is a maximum of $J$ at fixed $M$ and $\beta$. 
We now consider the stability of a gaseous system with an equation of state $p=p(\rho)$. The energy of this gaseous configuration is (Lynden-Bell \& Sanitt 1969)

$\mathcal{W}=\int \rho \int_{0}^{\rho} \frac{p\left(\rho^{\prime}\right)}{\rho^{\prime 2}} \mathrm{~d} \rho^{\prime} \mathrm{d}^{3} \boldsymbol{r}+\frac{1}{2} \int \rho \Phi \mathrm{d}^{3} \boldsymbol{r}$

We observe that the $J$-function (219) associated with a stellar system is equal (up to a negative proportionality factor $-\beta$ ) to the energy (223) of the corresponding barotropic gas. The stability theorem of Chandrasekhar for gaseous spheres requires that $\delta^{2} \mathcal{W} \geq 0$ (see Binney \& Tremaine 1987). This leads to the inequality (220) and to the eigenvalue Eq. (222). We now consider the dynamical stability of a self-gravitating gaseous system with respect to the Navier-Stokes or Euler equations (Jeans problem). This problem can be solved explicitly for isothermal and polytropic spheres (Chavanis 2002a,b). Adapting the same procedure for a general barotropic equation of state and denoting by $\lambda$ the growth rate of the perturbation (such that $\delta \rho \sim \mathrm{e}^{\lambda t}$ etc.), we obtain the eigenvalue equation

$\left[\frac{\mathrm{d}}{\mathrm{d} r}\left(\frac{p^{\prime}(\rho)}{4 \pi \rho r^{2}} \frac{\mathrm{d}}{\mathrm{d} r}\right)+\frac{G}{r^{2}}\right] q_{\lambda}(r)=\frac{\lambda^{2}}{4 \pi \rho r^{2}} q_{\lambda}(r)$,

with appropriate boundary conditions (see Appendix C). This equation is equivalent to the Eddington (1926) equation of pulsation but it proved to be more convenient in our previous investigations. We observe that Eqs. (222) and (224) are similar and that they coincide for marginal stability. This first demonstates the equivalence between the stability criterion of Eddington based on the equation of pulsation (224) and the stability criterion of Chandrasekhar based on the minimization of the energy (223). This also shows the equivalence between the maximization of the $J$-function for a stellar system at fixed $\beta$ and $M$ and the dynamical stability (with respect to the JeansEuler equations) of the corresponding barotropic star. Note that we have only studied the dynamical stability of barotropic stars with respect to small perturbations. However, we conjecture that the maximization of $J$ at fixed $M$ and $\beta$ is a condition of nonlinear dynamical stability for the Jeans-Euler equation. The proof is expected to be similar to the corresponding one for the Vlasov equation.

\subsection{A new interpretation of Antonov's first law}

We can now combine the previous results in order to provide a new interpretation of Antonov's first law (Binney \& Tremaine 1987 ) based on a thermodynamical analogy. We have seen in Sect. 7.7 that a stellar system with $f=f(\epsilon)$ and $f^{\prime}(\epsilon)<0$ is dynamically stable (nonlinearly) with respect to collisionless perturbations if $f$ is a maximum of a $H$-function $S[f]$ at fixed $E$ and $M$. According to the discussion following Eqs. (204) and (205), a sufficient condition of dynamical stability is that $f$ is a maximum of the $J$-function $J[f]$ at fixed $\beta$ and $M$. Now, according to Sect. 7.8, this is equivalent to the dynamical stability of the corresponding barotropic gas. Therefore, we come to the conclusion that "a stellar system is dynamically stable whenever the corresponding barotropic star is stable", which is Antonov's first law. Owing to the thermodynamical analogy, Antonov's first law is similar to the property that "canonical stability implies microcanonical stability" in thermodynamics. We note that the reciprocal of Antonov's first law is not true in general. If a stellar system is stable this does not necessarily imply that the corresponding barotropic gas is stable. Similarly, in thermodynamics, microcanonical stability does not necessarily imply canonical stability. This is the case only if the statistical ensembles are equivalent. We know, however, that this equivalence is broken for self-gravitating systems.

Let us illustrate these results in the case of polytropes. Taruya \& Sakagami (2002a, b) have investigated the stability of stellar systems in the framework of Tsallis generalized thermodynamics (see also Sect. 5.2). Due to the thermodynamical analogy discussed in Sects. 7.7 and 7.8, the study of Taruya $\&$ Sakagami can be used to determine the dynamical stability of collisionless stellar polytropes and polytropic gaseous spheres. In the generalized thermodynamical approach, it is found that the statistical ensembles are inequivalent. This implies that the dynamical stability of stellar polytropes and gaseous polytropes will also be inequivalent (Chavanis 2002b). Self-gravitating systems described by Tsallis entropy are thermodynamically stable in the microcanonical ensemble if $n<5$ or if the density contrast is sufficiently low for $n>5$. In that case, they are maxima of $S_{q}[f]$ at fixed $M$ and $E$. According to Sect. 7.7, this implies that stellar polytropes are dynamically stable solutions of the Vlasov equation for $n<5$ (and if the density contrast is sufficiently low for $n>5$ ). On the other hand, self-gravitating systems described by Tsallis entropy are thermodynamically stable in the canonical ensemble if $n<3$ or if the density contrast is sufficiently low for $n>3$. In that case, they are maxima of $J_{q}[f]$ at fixed $M$ and $\beta$. According to Sect. 7.8, this implies that polytropic gaseous spheres are dynamically stable solutions of the Jeans-Euler equations if, and only, if $n<3$ (or if, and only, if the density contrast is sufficiently low for $n>3$ ). We note, in particular, that gaseous polytropes with $3<n<5$ are unstable for sufficiently high density contrasts (e.g., complete polytropes) while corresponding stellar polytropes are stable. This shows that the reciprocal of Antonov's first law is wrong for polytropes. Due to the thermodynamical analogy, this can be related to an inequivalence of statistical ensembles in a region of negative specific heats (Chavanis 2002b). Our present study shows that the results obtained for isothermal stellar systems and stellar polytropes can be generalized to any $H$-function.

\section{Conclusion}

We have performed an exhaustive study of the thermodynamics of self-gravitating systems in various ensembles. This paper completes previous investigations on the subject and all ensembles have now been treated. Contrary to ordinary (extensive) systems, we have to perform a specific study in each ensemble since the stability limits differ from one to the other. Remarkably, the thermodynamical stability problem can be studied analytically or with simple graphical constructions. The dynamical stability of isothermal gaseous spheres can also be studied analytically both in Newtonian mechanics for the Euler-Jeans equations (Chavanis 2002a) and in general relativity for the Einstein equations (Chavanis 2002d). 
These results can be of relevance both for astrophysicists and statistical mechanicians and could make a bridge between these two communities. On an astrophysical point of view, they show that we must be careful to precisely define the ensemble in which we work since they are not equivalent. This does not affect the structure of the equilibrium configuration but it may affect its stability. On a physical point of view, this study fills an important gap in the statisical mechanics literature since the case of self-gravitating systems is not discussed at all in standard textbooks of statistical mechanics and thermodynamics.

We have also discussed the relevance of Tsallis generalized thermodynamics for stellar systems. Collisionless stellar systems such as elliptical galaxies can achieve a metaequilibrium state as a result of a violent relaxation. The Boltzmann entropy $S_{\mathrm{B}}[\bar{f}]$ is the correct entropy for these systems (in a coarsegrained sense and assuming that the system is non-degenerate). It measures the disorder where the disorder is equal to the number of microstates consistent with a given macrostate. However, $S_{\mathrm{B}}[\bar{f}]$ is not maximized by the system because of incomplete relaxation (this is independant on the fact that $S_{\mathrm{B}}[\bar{f}]$ has no maximum!). In any case, the state resulting from incomplete violent relaxation is a nonlinearly stable solution of the Vlasov equation (on a coarse-grained scale). If $\bar{f}=f(\epsilon)$, it maximizes a H-function $S[f]=-\int C(f) \mathrm{d}^{3} \boldsymbol{r} \mathrm{d}^{3} \boldsymbol{v}$, where $C(f)$ is a convex function, at fixed mass and energy. Therefore, we can use a thermodynamical analogy to study the dynamical stability of collisionless stellar systems. Tsallis entropies $S_{q}[f]$ are a particular class of $H$-functions leading to stellar polytropes. Stellar polytropes do not give a good fit of elliptical galaxies. A better model of incomplete violent relaxation, motivated by precise physical arguments, consists of an isothermal core and a polytropic halo with index $n \simeq 4$ (Hjorth \& Madsen 1993). This can be fitted by a composite Tsallis (polytropic) model with $q=1$ in the core (complete mixing) and $q \simeq 7 / 5$ in the envelope (incomplete mixing). More generally, the state resulting from incomplete violent relaxation does not necessarily maximize a $H$-function at fixed mass and energy. Due to the Jeans theorem, the distribution function can depend on other integrals than the stellar energy or the Jacobi energy. It is possible, nevertheless, that relevant distribution functions arising from incomplete violent relaxation maximize a $H$-function at fixed mass, energy, angular momentum and additional (adiabatic) invariants.

Acknowledgements. I am grateful to J. Katz and T. Padmanabhan for stimulating discussions and encouragement.

\section{Appendix A: Some useful identities for isothermal spheres}

Using the Emden equation (14), we have

$\int_{0}^{\alpha} \xi^{2} \mathrm{e}^{-\psi} \mathrm{d} \xi=\int_{0}^{\alpha}\left(\xi^{2} \psi^{\prime}\right)^{\prime} \mathrm{d} \xi=\alpha^{2} \psi^{\prime}(\alpha)=\alpha v_{0}$,

which establishes Eq. (67). On the other hand, using an integration by parts, we obtain

$$
\begin{aligned}
\int_{0}^{\alpha} \psi^{\prime} \xi^{3} \mathrm{e}^{-\psi} d \xi & =-\int_{0}^{\alpha} \xi^{3} \frac{\mathrm{d}}{\mathrm{d} \xi}\left(\mathrm{e}^{-\psi}\right) \mathrm{d} \xi \\
& =-\alpha^{3} \mathrm{e}^{-\psi(\alpha)}+\int_{0}^{\alpha} 3 \xi^{2} \mathrm{e}^{-\psi} \mathrm{d} \xi
\end{aligned}
$$

Combining with Eq. (A.1) and introducing the Milne variables, we establish Eq. (68). To establish Eq. (69), we start from the identity

$$
\begin{aligned}
& \int_{0}^{\alpha} \frac{\xi^{2} \psi^{\prime}}{\xi} \frac{\mathrm{d}}{\mathrm{d} \xi}\left(\xi^{2} \psi^{\prime}\right) \mathrm{d} \xi=\alpha^{3} \psi^{\prime}(\alpha)^{2} \\
& -\int_{0}^{\alpha} \frac{\xi^{2} \psi^{\prime}}{\xi} \frac{\mathrm{d}}{\mathrm{d} \xi}\left(\xi^{2} \psi^{\prime}\right) \mathrm{d} \xi+\int_{0}^{\alpha} \xi^{2} \psi^{\prime 2} d \xi
\end{aligned}
$$

which results from a simple integration by parts. Thus,

$$
\int_{0}^{\alpha} \xi^{2} \psi^{\prime 2} \mathrm{~d} \xi=-\alpha^{3} \psi^{\prime}(\alpha)^{2}+2 \int_{0}^{\alpha} \psi^{\prime} \xi^{3} \mathrm{e}^{-\psi} \mathrm{d} \xi
$$

where we have used the Emden Eq. (14). Combining this relation with Eq. (A.2), we obtain

$$
\int_{0}^{\alpha} \xi^{2} \psi^{\prime 2} \mathrm{~d} \xi=\alpha v_{0}\left(6-v_{0}-2 u_{0}\right)
$$

Now,

$$
\begin{aligned}
\int_{0}^{\alpha} \xi^{2} \psi \mathrm{e}^{-\psi} \mathrm{d} \xi & =\int_{0}^{\alpha} \psi\left(\xi^{2} \psi^{\prime}\right)^{\prime} \mathrm{d} \xi \\
& =\alpha^{2} \psi(\alpha) \psi^{\prime}(\alpha)-\int_{0}^{\alpha} \xi^{2} \psi^{\prime 2} \mathrm{~d} \xi
\end{aligned}
$$

From Eqs. (A.5) and (A.6) results Eq. (69).

\section{Appendix B: Some useful identities for polytropic spheres}

Using integrations by parts similar to those performed in Appendix A, we can easily establish the identities

$$
\begin{aligned}
& \int_{0}^{\alpha}\left(\theta^{\prime}\right)^{2} \xi^{2} \mathrm{~d} \xi=\frac{\alpha^{3} \theta^{\prime}(\alpha)^{2}}{5-n}\left(n+1+2 \frac{u_{0}}{v_{0}}-\frac{6}{v_{0}}\right) \\
& \int_{0}^{\alpha} \theta^{n+1} \xi^{2} \mathrm{~d} \xi=\frac{\alpha^{3} \theta^{\prime}(\alpha)^{2}}{5-n}\left(n+1+2 \frac{u_{0}}{v_{0}}-\frac{n+1}{v_{0}}\right), \\
& \int_{0}^{\alpha} \theta^{n} \theta^{\prime} \xi^{3} \mathrm{~d} \xi=\frac{\alpha^{3} \theta^{\prime}(\alpha)^{2}}{5-n}\left(-3-\frac{u_{0}}{v_{0}}+\frac{3}{v_{0}}\right) \\
& \int_{0}^{\alpha} \theta^{n} \xi^{2} \mathrm{~d} \xi=-\alpha^{2} \theta^{\prime}(\alpha) .
\end{aligned}
$$

\section{Appendix C: Equation of pulsations and boundary conditions}

In this paper and previous ones, we have studied the dynamical stability of barotropic gaseous spheres with the equation of pulsation

$\left[\frac{\mathrm{d}}{\mathrm{d} r}\left(\frac{p^{\prime}(\rho)}{4 \pi \rho r^{2}} \frac{\mathrm{d}}{\mathrm{d} r}\right)+\frac{G}{r^{2}}\right] q_{\lambda}(r)=\frac{\lambda^{2}}{4 \pi \rho r^{2}} q_{\lambda}(r)$,

where $\lambda$ is the growth rate of the perturbation (such that $\delta \rho \sim$ $\mathrm{e}^{\lambda t}, \delta v \sim \mathrm{e}^{\lambda t}$ etc.) and $q(r) \equiv \delta M(r)=\int_{0}^{r} \delta \rho 4 \pi r^{2} \mathrm{~d} r$ represent the mass perturbation within the sphere of radius $r$ which is related to the velocity by (Chavanis 2002a)

$\delta v=-\frac{\lambda}{4 \pi \rho r^{2}} q$. 
Obviously $q(0)=0$ and more precisely $q \sim r^{3}$ for $r \rightarrow 0$. If the system is confined within a sphere of fixed radius $R$, the conservation of mass imposes $q(R)=0$. This can also be obtained from Eq. (C.2) if we set $\delta v(R)=0$. If the system is free and the density vanishes at its surface, i.e. $\rho(R)=0$, like for a complete polytrope, then Eq. (C.2) again implies that $q(R)=0$ (Chavanis $2002 b)$. If, finally, the gaseous sphere supports a fixed external pressure (isobaric ensemble) the boundary condition requires that the Lagrangian derivative of the pressure vanishes at the surface of the configuration, i.e.

$\frac{\mathrm{d}}{\mathrm{d} t} \delta p+\delta v \frac{\mathrm{d} p}{\mathrm{~d} r}=0, \quad$ at $r=R$.

Using $\mathrm{d} \delta p / \mathrm{d} t=\lambda \delta p$ and Eqs. (C.2) and (122), we get for an arbitrary barotropic equation of state

$\frac{\mathrm{d} q}{\mathrm{~d} r}-\frac{q}{\rho} \frac{\mathrm{d} \rho}{\mathrm{d} r}=0, \quad$ at $r=R$.

If we set $\delta v=\mathrm{d} \delta r / \mathrm{d} t=\lambda \delta r$ and introduce the Lagrangian displacement $\xi(r) \equiv \delta r / r=\delta v / \lambda r$, we can easily check that Eq. (C.1) is equivalent to the Eddington (1926) equation of pulsations

$\frac{\mathrm{d}}{\mathrm{d} r}\left(p \gamma r^{4} \frac{\mathrm{d} \xi}{\mathrm{d} r}\right)+\left\{r^{3} \frac{\mathrm{d}}{\mathrm{d} r}[(3 \gamma-4) p]-\lambda^{2} \rho r^{4}\right\} \xi=0$,

where $\gamma(r) \equiv \mathrm{d} \ln p / \mathrm{d} \ln \rho$. The boundary condition at the origin is $\xi^{\prime}(0)=0$. On the other hand, if the system is enclosed within a box $\xi(R)=0$. Finally, if the pressure at the surface of the sphere is constant (possibly zero), the boundary condition (C.4) becomes

$\frac{\mathrm{d} \xi}{\mathrm{d} r}=-\frac{3 \xi}{r}, \quad$ at $r=R$.

\section{References}

Antonov, V. A. 1962, Vest. Leningr. Gos. Univ., 7, 135

Aronson, E. B., \& Hansen, C. J. 1972, ApJ, 177, 145

Bettwieser, E., \& Sugimoto, D. 1984, MNRAS, 208, 493

Bilic, N., \& Viollier, R. D. 1997, Phys. Lett. B, 408, 75

Binney, J., \& Tremaine, S. 1987, Galactic Dynamics (Princeton Ser. in Astrophysics)

Bonnor, W. B. 1956, MNRAS, 116, 351

Chandrasekhar, S. 1942, An introduction to the theory of stellar Structure (Dover)

Chavanis, P. H. 1998, MNRAS, 300, 981

Chavanis, P. H. 2002a, A\&A, 381, 340

Chavanis, P. H. 2002b, A\&A, 386, 732

Chavanis, P. H. 2002c, PRE, 65, 056123

Chavanis, P. H. 2002d, A\&A, 381, 709

Chavanis, P. H. 2002e, Statistical mechanics of two-dimensional vortices and stellar systems, in Dynamics and thermodynamics of systems with long range interactions, ed. T. Dauxois, S. Ruffo, E. Arimondo, \& M. Wilkens, Lecture Notes in Physics, vol. 602 (Springer) [cond-mat/0212223]
Chavanis, P. H. 2002f, Statistical mechanics of violent relaxation in stellar systems, in Proc. of the Conf. on Multiscale Problems in Science and Technology (Springer) [astro-ph/0212205]

Chavanis, P. H. 2002g, The self-gravitating Fermi gas, in Proceedings of the Fourth International Heidelberg Conference on Dark Matter in Astro and Particle Physics, ed. Klapdor-Kleingrothaus (NewYork: Springer) [astro-ph/0205426]

Chavanis, P. H. 2002h, PRE, submitted [cond-mat/0209096]

Chavanis, P. H., \& Ispolatov, I. 2002, PRE, 66, 036109

Chavanis, P. H., \& Sommeria, J. 1998, MNRAS, 296, 569

Chavanis, P. H., Sommeria, J., \& Robert, R. 1996, ApJ, 471, 385

Chavanis, P. H., Rosier, C., \& Sire, C. 2002, PRE, 66, 036105

Cohn, H. 1980, ApJ, 242, 765

de Vega, H. J., Sanchez, N., \& Combes, F. 1998, ApJ, 500, 8

de Vega, H. J., \& Sanchez, N. 2002, Nucl. Phys. B, 625, 409

Doremus, J. P., Feix, M. R., \& Baumann, G. 1971, Phys. Rev. Lett., 26,725

Ebert, R. 1955, Z. Astrophys., 37, 217

Eddington, A. S. 1926 The internal constitution of stars (Cambridge University Press)

Hawking, S. W. 1974, Nature, 248, 30

Hénon, M. 1961, Ann. Astrophys., 24, 369

Hertel, P., \& Thirring, W. 1971, in Quanten und Felder, ed. H. P. Dürr (Vieweg, Braunschweig)

Hjorth, J., \& Madsen, J. 1993, MNRAS, 265, 237

Horwitz, G., \& Katz, J. 1978, ApJ, 222, 941

Inagaki, S., \& Lynden-Bell, D. 1983, MNRAS, 205, 913

Ipser, J. R. 1974, ApJ, 193, 463

Ipser, J. R., \& Horwitz, G. 1979, ApJ, 232, 863

Jaffe, W. 1987, in Structure and dynamics of elliptical galaxies, Proc. IAU Symp, 127, ed. T. de Zeeuw (Reidel, Dordrecht)

Kandrup, H. E. 1981, ApJ, 244, 316

Katz, J. 1978, MNRAS, 183, 765

Katz, J. 1980, MNRAS, 190, 497

Katz, J., \& Okamoto, I. 2000, MNRAS, 371, 163

Kiessling, M. 1989, J. Stat. Mech., 55, 203

Lecar, M., \& Katz, J. 1981, ApJ, 243, 983

Lee, E. P. 1968, ApJ, 151, 687

Lynden-Bell, D. 1967, MNRAS, 136, 101

Lynden-Bell, D., \& Eggleton, P. P. 1980, MNRAS, 191, 483

Lynden-Bell, D., \& Sanitt, N. 1969, MNRAS, 143, 167

Lynden-Bell, D., \& Wood, R. 1968, MNRAS, 138, 495

McCrea, W. H. 1957, MNRAS, 117, 562

Michel, J., \& Robert, R. 1994, Commun. Math. Phys., 159, 195

Ogorodnikov, K. F. 1965, Dynamics of stellar systems (Pergamon)

Padmanabhan, T. 1989, ApJS, 71, 651

Padmanabhan, T. 1990, Phys. Rep., 188, 285

Padmanabhan, T. 1991, MNRAS, 253, 445

Penston, M. V. 1969, MNRAS, 144, 425

Plastino, A., \& Plastino, A. R. 1997, Phys. Lett. A, 226, 257

Plummer, H. C. 1911, MNRAS, 71, 460

Sire, C., \& Chavanis, P. H. 2002, PRE, 66, 046133

Taruya, A., \& Sakagami, M. 2002a, Physica A, 307, 185

Taruya, A., \& Sakagami, M. 2002b, Physica A, submitted [cond-mat/0204315]

Terletsky, Y. P. 1952, Zh. Eksper. Teor. Fiz., 22, 506

Tremaine, S., Hénon, M., \& Lynden-Bell, D. 1986, MNRAS, 227, 543

Tsallis, C. 1988, J. Stat. Phys., 52, 479

Yabushita, S. 1968, MNRAS, 140, 109 